\title{
Investigating the effect of temporal and spatial flexibility on the performance of one-way electric carsharing systems
}

\author{
Burak Boyac1 $^{\mathrm{a}, *}$, Konstantinos G. Zografos ${ }^{\mathrm{a}}$ \\ ${ }^{a}$ Centre for Transport and Logistics (CENTRAL), Lancaster University Management School, Lancaster, LA1 4YX, UK
}

\begin{abstract}
One-way electric carsharing systems provide an environmentally friendly option for facilitating urban mobility needs. However, the management of one-way electric carsharing systems presents operational challenges stemming from the need to relocate cars in order to strike an optimum balance between demand and supply. As a result, the cost associated with vehicle relocation operations represents a significant proportion of the total operating cost. The introduction of temporal and spatial flexibility regarding the pick-up and drop-off of vehicles provides the means of improving the efficiency of one-way electric carsharing systems. However, the literature currently lacks models that can be used to investigate the effect of temporal and spatial flexibility on the performance of one-way electric carsharing systems. In this paper, we are introducing an integrated modeling and solution framework for investigating the effect of temporal and/or spatial flexibility, and different reservation options to the profitability and utilization of one-way electric carsharing systems. The application of the proposed framework to a realistic size system suggests that spatial flexibility has a stronger effect on the system performance than temporal flexibility. Furthermore, both spatial and temporal flexibility can increase the profitability of the system by serving more customers with fewer vehicle relocation needs.
\end{abstract}

Keywords: one-way carsharing, vehicle relocation optimization, integer programming, network flow

\section{Introduction}

In 2014, more than 54\% of the world population was living in urban areas. By 2060 it is expected this ratio will go up to 66\% (UN, 2014). In order to deal with increased population with limited resources, local and regional governments try to decrease private-use vehicles with disincentives such as congestion-pricing and increased taxation. Although these measures encourage some city dwellers to choose more societal-friendly options, there is still a significant proportion of trips that are unavoidably require driving a vehicle. One of the environmental-friendly options of having the option of providing mobility on demand without owning one is using a shared-use vehicle system (e.g. bikesharing, carsharing) (Shaheen et al., 2015).

Carsharing is a more accessible and efficient car rental concept usually for a short period of time. Different than the conventional car rental, in a carsharing system, users have self-service access to vehicles scattered around the city throughout the day. Thanks to rapid improvements in the mobile communication technologies, users are provided with a contactless card and an dedicated app that allows them to locate and access vehicles. When someone becomes member of the service, he/she gains access to vehicles (in some cases located in multiple cities) without any time limitation in a short period of time.

Carsharing has various benefits to environment and society as a whole. By eliminating the cost of owning a car, it reduces the personal transportation cost, increases mobility to disadvantaged groups and encourages people to prefer more environmental-friendly alternatives. It decreases vehicle kilometers travelled, congestion and emissions eventually (Crane et al., 2012). Each shared-use vehicles removes 7-10 privately owned vehicles from the streets (Shaheen et al., 2015).

\footnotetext{
${ }^{*}$ Corresponding author

Email addresses: b. boyaci@lancaster.ac.uk (Burak Boyacı), k.zografos@lancaster.ac.uk (Konstantinos G. Zografos)
} 
Carsharing systems can be classified according to their operating practices. Station-based systems allow users to pick-up and drop-off cars to/from designated parking spots. In round-trip systems each vehicle has a designated parking spot and vehicles should be returned to their pick-up locations after their use. More flexible one-way systems allow users to pick-up and drop-off vehicles from/to different stations. Free-floating systems allow users to park the rented vehicles to any legal parking spots in a designated area. Carsharing systems can also be classified according to vehicle engine types, electric or non-electric. Although the former option with its zero emissions is more environmental friendly, it also needs investment in charging facilities and consideration of battery levels when making rental decisions. There is also a classification for reservation opportunities. Some carsharing systems allow only last-minute reservations, reserving vehicles for a period of time in the near future, usually between 15 minutes and an hour, whereas some other systems allow to have long-term reservations for the distant future, which could be a couple of hours to weeks before rental. In the former option, users are allowed to lock vehicles with an app for a period of time whereas in the latter option users are provided with an option to have reservations in the future for a period of time. Although users prefer to have more flexible one-way or free-floating options, operators are usually inclined to provide simple-to-operate round-trip systems. Specifically, the complexity and cost of relocation operations, repositioning vehicles with dedicated personnel to have distribution of vehicles and as a result available spots at the right place and time, is a big factor in this decision (Boyac1 et al., 2015, 2017).

Relocation operations are usually leading to higher operating costs, lower vehicle utilization rates, and larger fleets. One approach that can be used to reduce the operating cost of one-way carsharing systems is to use the carsharing system users to perform part of the required relocation operations (Barth et al., 2004). This means that potential users of the system may be offered vehicle pick-up and drop-off locations and/or times that are not necessarily their first choice (Correia et al., 2014). The introduction of temporal and/or spatial flexibility provides operators of oneway electric carsharing systems, the opportunity to better match the temporal and spatial profile of demand with the required supply, leading to improved system performance.

In this paper, we are focusing on the operational decisions in one-way station-based electric carsharing systems. In particular, we investigate the effect of spatial and temporal flexibility, and different reservation options to the profitability and utilization of one-way electric carsharing systems. Our objective is to develop a modelling and solution framework that will provide operators of one-way electric carsharing systems the capability to consider simultaneously the temporal and spatial flexibility of reservations and relocation operations. The proposed framework will help decision makers to assess the impact of different levels of flexibility on the economic performance of their system.

The proposed framework contribute to the current state-of-the art by introducing following concepts:

1. Users are offered trips that are slightly different than what they requested for a reduced price. We limit the difference with predefined maximum spatial $(0-1 \mathrm{~km})$ and temporal (0-60 minutes) flexibility levels.

2. The introduction of a clustering algorithm using asymmetric travel times. The proposed clustering procedure improved the computational performance of our model, through the grouping of carsharing stations, without compromising the accuracy of the model results.

3. The Charging level feasibility problem is checked by a mathematical model in addition to a simulator. The new joint personnel and vehicle flow model explores the entire solution space and finds feasible schedules in many instances at which simulation cannot.

We organize the rest of the paper as follows: Section 2 covers the most recent literature review that is relevant to our research. We conclude this section with the rationale for the need of this research. Section 3 presents the solution framework. We describe all relevant models and approaches used in the development of the framework in this section. Section 4 provides the implementation details and results of the computational experiments. Section 5 discusses the concluding remarks and future research directions.

\section{Literature review}

In this work, we build an optimization framework for the operational decisions (e.g. accepting/rejecting demand requests, relocating vehicles and personnel) of one-way electric carsharing systems that provides spatial and temporal flexibility to its customers. In what follows, we are focusing our attention to previous work that has modelled operational decisions of one-way (electric) carsharing systems. The list of papers reviewed are also given in Table 1. 
Table 1: List of referenced articles

\begin{tabular}{|c|c|c|c|c|c|}
\hline Article & $\begin{array}{c}\text { one-way } \\
\text { trips }\end{array}$ & $\begin{array}{c}\text { electric } \\
\text { vehicles }\end{array}$ & $\begin{array}{c}\text { relocation } \\
\text { operations }\end{array}$ & $\begin{array}{c}\text { personnel } \\
\text { movements }\end{array}$ & $\begin{array}{c}\text { temporal and spatial } \\
\text { flexibility }\end{array}$ \\
\hline Nair and Miller-Hooks (2011) & $\checkmark$ & $\times$ & $\checkmark$ & $\times$ & $\times$ \\
Bruglieri et al. (2014) & $\checkmark$ & $\checkmark$ & $\checkmark$ & $\checkmark$ & $\times$ \\
Correia et al. (2014) & $\checkmark$ & $\times$ & $\times$ & $\times$ & $\times$ \\
Nourinejad et al. (2015) & $\checkmark$ & $\times$ & $\checkmark$ & $\checkmark$ & $\times$ \\
Repoux et al. (2015) & $\checkmark$ & $\times$ & $\checkmark$ & $\checkmark$ & $\times$ \\
Boyaci et al. (2017) & $\checkmark$ & $\checkmark$ & $\checkmark$ & $\checkmark$ & $\times$ \\
Gambella et al. (2018) & $\checkmark$ & $\checkmark$ & $\checkmark$ & $\checkmark$ & $\checkmark$ \\
Xu et al. (2018) & $\checkmark$ & $\checkmark$ & $\checkmark$ & $\checkmark$ & $\times$ \\
Zhao et al. (2018) & $\checkmark$ & $\checkmark$ & $\checkmark$ & $\checkmark$ & $\times$ \\
? & $\times$ & $\times$ & $\times$ & $\times$ & $\checkmark$ \\
? & $\checkmark$ & $\checkmark$ & $\checkmark$ & $\checkmark$ & \\
proposed framework & $\checkmark$ & $\checkmark$ & $\checkmark$ & $\checkmark$ & \\
\hline
\end{tabular}

For a comprehensive review of carsharing papers, users are referred to the survey papers Jorge and Correia (2013), Brandstätter et al. (2016), Ferrero et al. (2018) and Laporte et al. (2018).

Nair and Miller-Hooks (2011) formulate the operational problem as a mixed integer stochastic optimization problem. The model aims to find the ideal vehicle locations that serves a number of demand requests of the near future with minimum operational cost. The model is tested on a system with 94 vehicles and 14 stations operating in Singapore.

Bruglieri et al. (2014) formulate and electric vehicle relocation problem in a station-based one-way carsharing system. They propose to use bikes to travel between stations then repositioning vehicles between stations. They consider the projected travel demand in their analysis and assume all demand must be served. They tested their model on a system with 30 electric vehicles.

Correia et al. (2014) propose a mathematical model to evaluate the impact of user flexibility and sharing vehicle stock information with the users in a one-way carsharing system. They test three different scenarios: inflexible users that use only closest stations to their origin and destination, flexible users that are content to use second and third closest stations as alternatives without vehicle and empty spot information and flexible users with adequate system information. Their model has not considered relocation decisions, flexibility in pick-up times and the discounts in case of offering alternative trip options. They tested the algorithm on a system with 116 potential locations with station sizes up to 35 spots. Results show that the flexibility with and without option increase the profit by around 3 and 5 fold compared to the scenario without flexibility.

Nourinejad et al. (2015) deal with vehicle and staff relocations with two integrated multiple travelling salesman formulations. They use mathematical formulation to solve instances with 40 demand requests. For larger instances, a heuristic approach is developed by decomposing the first formulation to two formulations and solving them sequentially.

Repoux et al. (2015) develop an event based simulator for the analyses of the algorithms in a station-based oneway carsharing system. It uses limited information (instead of the full information) and the idea of partial floating (if the stations are full vehicles are allowed to park outside designated parking spots). They implemented an optimization framework that decides on relocations to perform and the personnel to do relocations in separate mathematical models. Tested on a system with 59 stations and 59 to 118 vehicles.

Gambella et al. (2018) consider the charging level of the vehicles in the mathematical model. The first model is used to find the set of served trips and relocation operations while considering the detailed personnel movements. The model also decides on the optimal locations at the beginning of the day. The secondary model is utilized to find the best relocation strategy during the night when the system is not available to users. It is found that the mathematical model is computationally impractical and as a result a few heuristic approaches are used instead. The system is tested on a system with 14 stations and 20 electric vehicles for a 10 hour period (i.e. 40 intervals of 15 minutes). 
$\mathrm{Xu}$ et al. (2018) consider the fleet sizing and trip pricing problems together while considering vehicle and personnel relocation operations and elastic demand. They propose a mixed integer non-convex programming problem formulation. They use outer approximation method to solve problem optimally. They tested the algorithm on a system with 27 stations with 500 spots each and 5000 electric vehicles. Since they use pricing and elastic demand, the number of aggregated served trip goes up to 268 among 39787 potential requests (when the system is free of charge).

Zhao et al. (2018) deal with the vehicle and staff relocations simultaneously. They propose a mathematical model working on discrete time-space. They do not take station capacities into consideration and assume all requests are served. They minimize the cost of vehicles, personnel and relocations in the objective function and use Lagrangean relaxation to find fast near-optimal solutions. They tested the algorithm on instances with 30 stations, 60 trip requests and stations and 30 relocation personnel.

? considers temporal and spatial flexibility in round-trip carsharing systems. They propose a mathematical model and solve it to optimality for weekly instances. They observe that the spatial flexibility of $1 \mathrm{~km}$ can decrease the fleet size (12\%) two times more than the temporal flexibility of 4 hours (4\%). They also observe synergy happens when users accept both temporal and spatial flexibility to a range together. They also evaluate the cost of flexibility to customers with the help of over 1500 responds.

? consider relocation operations and use Adaptive Large Neighborhood Search Algorithm. This algorithm does not decide one accepting/rejecting demand but instead assumes every demand requested is served. They aimed to minimize the number of relocation personnel operating the system. The algorithm is tested on instances on the systems with up to 100 pick-up and drop-off requests in total. Their further analyses has shown that in all small and in most of medium sized instances, they found the optimal solutions whereas in the large instances, the average optimality gap was around 6\%.

Our literature review (summarized in Table 1) revealed that the current state-of-the art lacks models that can investigate the effect of temporal and/or spatial flexibility on the performance of one-way electric carsharing system, while providing a realistic representation of the car and personnel relocation, and car charging operations.

\section{Methodology}

In this work, we are considering a reservation based one-way (station based) electric carsharing system with a flexibility of pick-up time, and origin and destination of trips. In what follows, we are presenting underlying the development of the proposed framework.

- The demand is known in advance. Although in some approaches, each demand set is revealed iteratively, the system does not start to fully operate before the entire demand is known to the system.

- The travel time between each station pairs is deterministic and constant throughout the day. The relocation time between any two stations equal to the travel time plus a fixed amount of time that is required to plug-off and on the vehicles.

- Each station has a fixed capacity of parking spots with standard chargers. As soon as vehicles arrive to the stations, either the users or relocation personnel connect vehicles to dedicated chargers.

- The relocation operations between stations are executed throughout the day by a dedicated team of relocation personnel. Each personnel works during one of the predefined working shifts. They travel between stations with (driving) or without (moving) vehicles. The speed of driving (i.e. personnel travelling with a vehicle between two stations) and moving (i.e. personnel travelling without a vehicle between two stations) operations can be different.

- The framework decides on the starting and ending nodes of each personnel and their detailed itinerary for their shifts. Although it can be altered by additional constraints, the current framework uses the optimal starting locations that maximizes the profit.

- The solution framework models the system with short and equal time intervals (e.g. 15 minutes). The vehicles and the relocation personnel cannot have more than one state during any time interval.

- The solution framework offers alternative trips that may differ from their requested and preferred choice. The alternative trips can start (and end) earlier or later than the requested time, originate from and/or destined to different stations. For the simplicity, we assume that the trip length and duration are not changing when an alternative is offered. Original and alternative requests have the same trip lengths and driving distances. 
The operator offers alternative trips with a discount depending on the difference between the start times', and the distances between original and destination stations' of alternative and original trip requests. There is an incentive for the operator to offer users their original requests. For simplicity and due to lack of user behaviour data regarding temporal and spatial flexibility in this paper, we use predefined maximum (pick-up) time and (origin and destination station) distance values for all users. If the flexibility regarding the users' behaviour is provided, the framework can be used without substantial changes to solve a problem with unique set of alternatives for every requests with predefined revenue.

- The framework maximizes the profit. The revenue of the system comes from serving the requested trips. Fuel cost of relocations and personnel hiring cost are deducted from the revenue to calculate the profit. Although it can be altered by additional constraints, number of personnel assigned to each shift is decided by the solution framework. Vehicle depreciation and station operating related costs are not taken into consideration since both the vehicle and station related properties are taken as input to the model.

- In order to make model easier to solve, the stations are partitioned into clusters and a representative node is introduced to each cluster. Instead of modelling relocation operations from origin to destination stations directly, we model relocations in three separate "imaginary" steps: (1) from the origin station to origin cluster node, (2) from the origin cluster node to destination cluster node, and (3) from the destination cluster node to destination station. Each step takes non-negative number of time steps. These clusters are decided by utilizing mathematical model and they never underestimate the real travel times.

- The travel times between two stations are not set to be symmetric. Travelling from stations $i$ to $l$ is not equal to travelling from station $l$ to $i$. This property is taken into consideration in every step of the solution framework.

- In order to make the solution framework more efficient, different approaches are used to solve the vehicle assignment problem. The proposed framework offers the capability to use either simulation or optimization for the vehicle assignment problem. After solving the Flexible Operational Optimization Model, the faster approach (i.e. simulation) that do not explore the entire solution space is used first. If a satisfactory solution is not found, we utilize a slower approach (i.e. optimization) that explore the entire solution space for the sake of efficiency.

The complete flow chart of the solution framework is given in Fig. 1. We start the problem with raw input data. We use the asymmetric distance matrices for both driving (i.e. relocation with a vehicle) and moving (i.e. relocation without a carsharing vehicle) operations in Assymmetric Station Clustering Model (ASCM, see Section 3.1). Since the locations of stations are the same for all instances, this model is solved twice, for driving and moving operations separately.

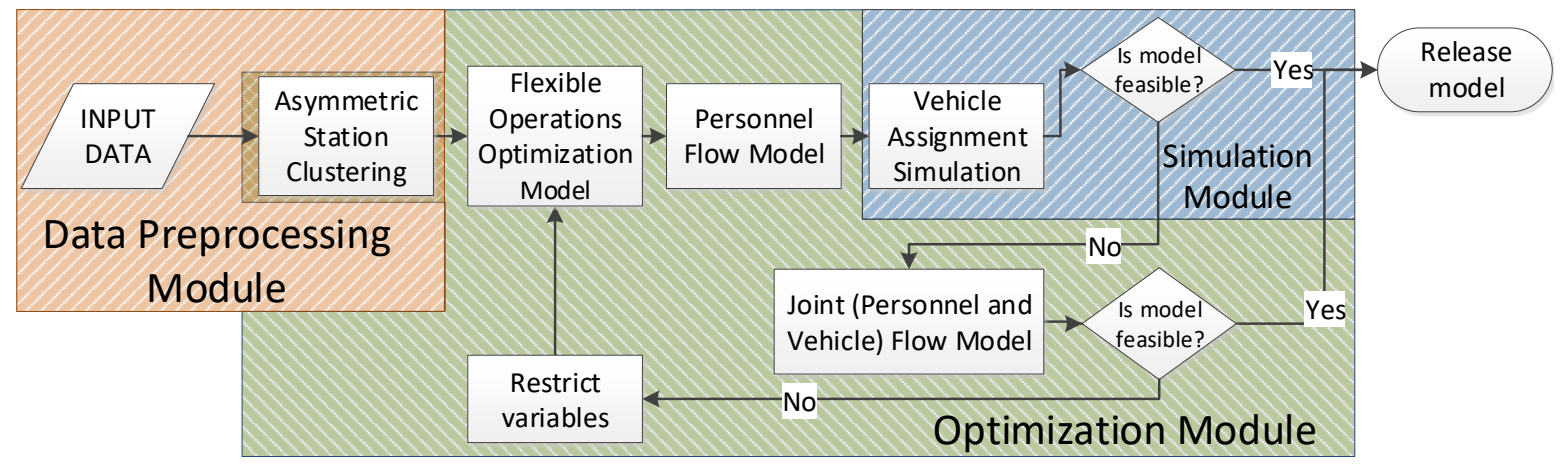

Figure 1: Proposed solution framework

After clusters are decided by ASCM, the Flexible Operations Optimization Model (FOOM, see Section 3.2) is solved with the input from raw data (e.g. demand requests, shift start and end times) and ASCM. The FOOM regards each personnel and vehicle movements as unit flows. It solves a parallel network flow problem that models vehicles and each personnel shift in separate networks as unit flows (see Fig. 2). The solution of the ASCM corresponds to more than one feasible vehicle and personnel assignments. Note that, for the electric carsharing systems, some or 
all of these assignments are not necessarily charging level feasible solutions, i.e. vehicles may reach charging levels lower than the level they are allowed to serve.
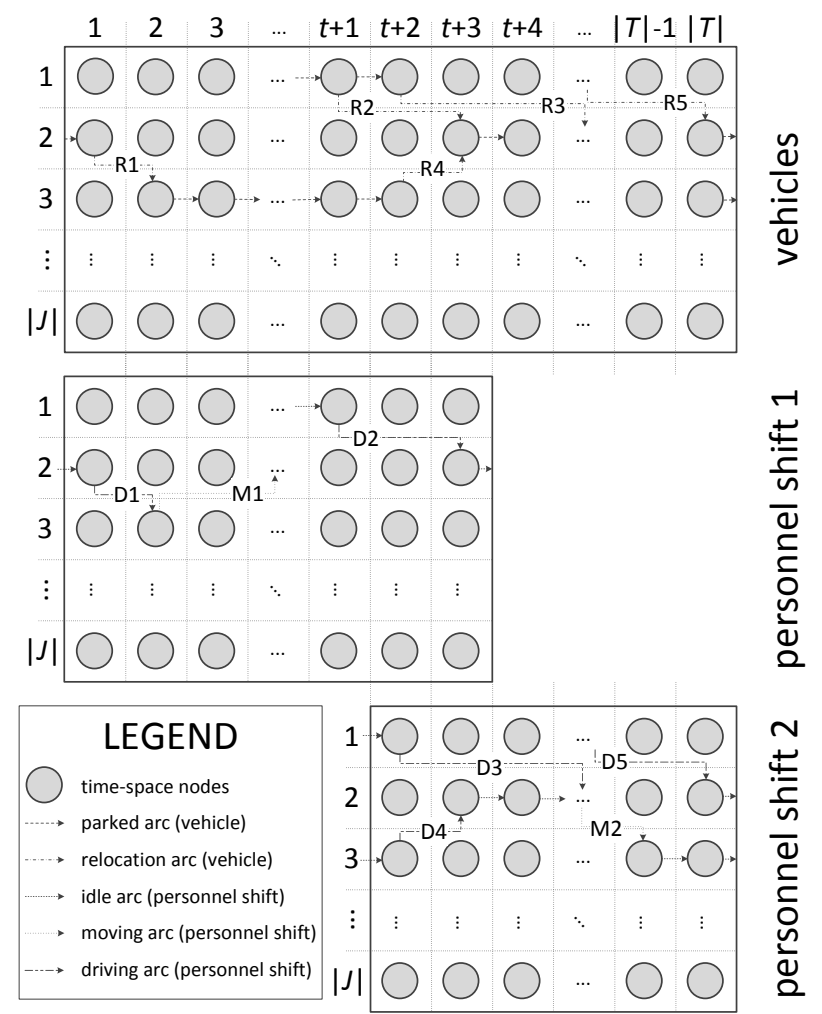

Figure 2: Time-space network figure from Boyac1 et al. (2017). Parallel time-space networks for vehicles and personnel shift for the set of stations (J) and time intervals (T). Each network have separate set of flows. In vehicle network, parked and relocation (R) flows are used. In the personnel network, we see flows for idle, moving (M) or driving (D) personnel. There is a corresponding relocation flow in the vehicle network for every driving flow that exists in one of the personnel networks.

The next step after solving the FOOM is finding personnel and vehicle flows. This problem is solved two different methods in the solution framework to improve the efficiency of the solution process. The Personnel Flow Model (PFM, see 3.4) considers only the flows of personnel and creates complete relocation assignments from the constituent elements of relocation flows, i.e. from origin to destination through origin and destination cluster nodes. The solution of the PFM identifies all relocation operations between two stations with and without vehicles. Then the Vehicle Assignment Simulation (VAS, see 3.5), a discrete event simulation that considers myopic assignment policies, is utilized. If it returns a charging level feasible schedule, then the result is released. If it is not feasible and there is at least one vehicle with an infeasible charging level, then we use the Joint (Personnel and Vehicle) Flow Model (JFM, 3.3). The JFM takes the solution of the latest FOOM as an input and decides on the personnel flows and vehicle assignments simultaneously within a single mathematical model. Since it explores a larger solution space than the previous approach, it takes longer to solve than the PFM and VAS combined. However, since it explores the entire solution space in one step, it may return a charging level feasible solution when the other approach cannot. If it finds out there is a charging level feasible solution, the solution is released and the algorithm terminates. If there does not exist any feasible solutions, the JFM releases the trips which were attempted by the vehicles with infeasible charging levels. The FOOM requires the vehicles that are serving these trips to be charged after (if) they are served. We resolve the FOOM and this loop continues until a charging level feasible solution is released.

In our solution framework, we have also introduced an another approach besides the VAS and the JFM. We call this model Vehicle Assignment Model (VAM, see Appendix A) since it uses the relocation assignments created by the PFM. Our expectation was that it would be more efficient than running the JFM. However our preliminary experiments have shown that the running times for the same instances of the VAM and JFM are almost the same. As a result, we 
remove this model from the solution framework.

In what follows we are providing the mathematical description of the constituent modules of the proposed framework.

\subsection{Asymmetric Station Clustering Model (ASCM)}

Asymmetric Station Clustering Model (ASCM) is used to cluster stations to simplify relocation operations in Flexible Operations Optimization Model (FOOM). If the time interval and node sets are represented with $T$ and $N$ simultaneously, allowing relocation operations throughout the day between any station pairs requires $O\left(|T||N|^{2}\right)$ variables for relocation operations only. In order to use fewer relocation variables, we introduce imaginary cluster nodes and make relocation operations in three steps (Boyac1 et al., 2017). When there is a relocation from node $j$ to $l$, the relocation is done (1) from node $j$ to the cluster node of $j$, then (2) from the cluster node of $j$ to cluster node of $l$, and finally (3) from the cluster node of $l$ to node $l$. If the cluster set is represented with $M$ this approach decreases number of variables for relocation to $O\left(|T||M|^{2}+|T||N|\right)$. Although the clustering approach slightly overestimates the relocation times between a few node pairs, it helps us to build a solution framework easier to solve. Note that, a similar clustering approach is used by Boyacı et al. (2017). However, in this paper, we create the clusters by solving a mathematical model and allow asymmetric travel times between node pairs. This difference makes the approximation of the travel times and eventually the solution gathered from the framework more accurate.

\subsubsection{Set and indices}

$j, l \in J$ nodes (stations)

$m, n \in M$ clusters

\subsubsection{Parameters}

$t_{j l}$ travel time from node $j$ to $l$

$w_{j l}$ weight of relocations from node $j$ to $l$ expressing the popularity (preference of users to use this station)

\subsubsection{Variables}

$x_{j}^{m} 1$ if node $j$ is in cluster $m, 0 \mathrm{o} / \mathrm{w}$

$u_{j}$ approximated travel time from node $j$ to its own cluster

$\bar{u}_{j}$ approximated travel time to node $j$ from its own cluster

$v^{m n}$ approximated travel time from cluster node $m$ to $n$

$d_{j l}$ approximated travel time from node $j$ to node $l$

\subsubsection{Formulation}

$$
\begin{aligned}
\min & \sum_{j, l} w_{j l}\left(d_{j l}-t_{j l}\right) \\
\text { s.t. } & \sum_{m} x_{j}^{m}=1 \\
& d_{j l} \leq u_{j}+v^{m n}+\bar{u}_{l}+\mathrm{M}\left(2-x_{j}^{m}-x_{l}^{n}\right) \\
& d_{j l} \geq u_{j}+v^{m n}+\bar{u}_{l}+\mathrm{M}\left(x_{j}^{m}+x_{l}^{n}-2\right) \\
& d_{j l} \geq t_{j l} \\
& \sum_{j} x_{j}^{m} \geq 1 \\
& x_{j}^{m} \in\{0,1\} ; u_{j}, \bar{u}_{j}, v^{m n}, d_{j l} \in\{0\} \cup \mathbb{Z}^{+}
\end{aligned}
$$$$
\forall j, l \neq j \in J ; m, n \in M(4)
$$

The clustering problem is formulated by Eqs. 1-7. The formulation aims to minimize the weighted error of relocation operations through clustering nodes. Inputs of the model are the number of clusters and the travel time between each station pairs. The solution of the model determines the clusters, and the approximated travel times 
(1) from nodes to clusters, (2) between clusters and (3) from clusters to nodes. Please note that the approximated travelling times through clusters cannot be shorter than the actual travel times. Therefore, this model prevents the underestimation of travel times between node pairs.

Objective 1 minimizes the weighted difference between the estimated travel time $\left(t_{j l}\right)$ and the real travel time $\left(d_{j l}\right)$ between every node pairs. By introducing weights in the objective function we aim to incorporate the "popularity" of the station in the clustering decisions. In the computational experiments, the weight used for every pair $\left(w_{j l}\right)$ is equal to the total number of trips originating from and destined to nodes $j$ and $l$. We assume, if a station is used more frequently by the users, it is more likely that it is involved in relocation operations.

Constraints 2 require that every node should belong to one and only one cluster.

Constraints 3 and 4 calculate the approximated travel time $d_{j l}$ between every node pairs $j$ and $l$. Constraints 3 state that if nodes $j$ and $l$ belong to clusters $m$ and $n$ respectively, then travel time $d_{j l}$ should be less than or equal to the summation of travel times (1) from node $j$ to its cluster node, (2) from cluster node $m$ to cluster node $n$ and (3) from the cluster node of $l$ to node $l$. If node $j$ does not belong to cluster $m$ or node $l$ does not belong to cluster $n$, the big-M part of the constraints make the inequalities not binding. Similarly, constraints 4 binds the value of $d_{j l}$ from above. Constraints 3 and 4 ensure that $d_{j l}=u_{j}+\bar{u}_{l}+v^{m n}$ if nodes $j$ and $l$ belong to clusters $m$ and $n$ respectively.

Constraints 5 require that between every node pairs, approximated travel time $\left(d_{j l}\right)$ to be at least as long as the the real travel time $\left(t_{j l}\right)$. These constraints prevent any approximated travel times to be smaller than the real travel times.

Constraints 6 postulate every cluster should at least have one node assigned to it. They are redundant constraints added to the model to have shorter solution times.

Constraints 7 set the domains of each variable. All the variables except $x_{j}^{m}$ can take any non-negative integer values whereas $x_{j}^{m}$ is defined as a binary variable.

\subsection{Flexible Operations Optimization Model (FOOM)}

The Flexible Operations Optimization Model (FOOM) formulates the operational planning of a carsharing system as an advanced parallel network flow problem (Fig. 2) The model considers accepting/rejecting each demand request, decides on the offered trip, which are either exactly the same as the requested demand or different but within maximum temporal and spatial flexibility, to the user, and determines the relocations with and without vehicles throughout the operational time of the system. In order to increase the efficiency of the model, relocations with and without vehicles are done through two different sets of clustering nodes (see Section 3.1), and each vehicle and personnel at each shift are considered as unit flows on different layers of the parallel networks. The unique flows for each vehicle and personnel is decided by different methods including the Joint (Personnel and Vehicle) Flow Model (JFM) given in Section 3.3. The FOOM uses an approach similar to the Operations Optimization Model described in Boyac1 et al. (2017). However, there are significant differences between the two models. This new model allows the operator to offer alternative trips different than the original requests with a discount. Furthermore, it utilizes asymmetric travel times between stations during relocations and utilizes relocation personnel better. Furthermore, the current formulation makes a core realistic assumption regarding the availability of the relocation personnel. Specifically in the previous formulation (see Boyac1 et al., 2017) an assumption was made requiring a relocation personnel to wait one time interval (15 minutes) before its assignment to a new relocation task. In this version we have lifted this constraint and therefore the relocation personnel can be assigned as soon as the previous request is completed. As a result, the utilization of the relocation personnel is improved.

\subsubsection{Sets and indices}

$i \in I$ demand

$j, l \in J$ stations

$t, u, w \in T$ time intervals

$s \in S$ working shifts

$b, d \in B$ station clusters

$e \in E$ start time options (of any demand)

$o, \bar{o} \in O$ destination/origin options (of any demand)

$a$ trip (4-tuple of demand, and start time, origin and destination options) 


\subsubsection{Parameters}

start $(s) / \operatorname{end}(s)$ start/end time intervals of shift $s$

$\operatorname{start}(i, e) / \operatorname{end}(i, e)$ pick-up/drop-off time of start time option $e$ of demand $i$

$\operatorname{origin}(i, e, \bar{o})$ origin option $\bar{o}$ of demand $i$

$\operatorname{dest}(i, e, o)$ destination option $o$ of demand $i$

c.end $(i, e)$ last charging time interval of option $e$ of demand $i$

d.end $(t, b, d)$ time interval at which the driving starting at $t$ reaches from cluster $b$ to $d$

m.end $(t, b, d)$ time interval at which the moving starting at $t$ reaches from cluster $b$ to $d$

d.from $(j)$ time interval difference between the driving starting from station $j$ and reaching to its cluster node

$\mathrm{m}$.from $(j)$ time interval difference between the moving starting from station $j$ and reaching to its cluster node

d.to $(j)$ time interval difference between the driving reaching to station $j$ starting from its cluster node

m.to $(j)$ time interval difference between the moving reaching to station $j$ starting from its cluster node

$\mathrm{PC}_{s}$ cost of relocation personnel for shift $s$

$\overline{\mathrm{RC}}_{j} / \mathrm{RC}_{j}$ intra-relocation cost from/to station $j$

$\widetilde{\mathrm{RC}}_{b d}$ inter-relocation cost from cluster $b$ to $d$

SG safety gap (i.e. number of time intervals vehicles forced to be kept at the station after drop-off)

$I_{c}$ set of trip options requiring charging

$\mathrm{P}_{i}$ gross revenue of serving demand $i$

$\tilde{\mathrm{D}}_{i}^{e} / \mathrm{D}_{i}^{o} / \overline{\mathrm{D}}_{i}^{\bar{o}}$ discount of demand $i$ if it is served by \{start time option $\left.e\right\} /$ \{origin option $\left.\bar{o}\right\} /$ \{drop-off option $o$ \}

$a_{i} / a_{e} / a_{o} / a_{\bar{o}}$ demand / \{start time option\} / \{origin option\} / \{destination option\} of tuple $a$

$C$ set of trips that need charging.

$C$ (a) set of recently added trips to the model that are served by the same vehicle which serves trip $a$

\subsubsection{Variables}

$z_{i} 1$ if demand $i$ is served; $0 \mathrm{o} / \mathrm{w}$

$\tilde{z}_{i}^{e} 1$ if demand $i$ 's start time option $e$ is served, $0 \mathrm{o} / \mathrm{w}$

$\bar{z}_{i}^{\bar{e} \bar{o}} / z_{i}^{e o} 1$ if demand $i$ 's \{origin option $\left.\bar{o}\right\} /\{$ destination option $o\}$ with time option $e$ is served; $0 \mathrm{o} / \mathrm{w}$

$\hat{z}_{i}^{e \bar{o} o} 1$ if demand $i$ 's time option $o$, origin option $\bar{o}$, and destination option $o$ is served and this trip is in the forced charging set $(C) ; 0 \mathrm{o} / \mathrm{w}$

$v_{s}$ number of personnel used from shift $s$

$n_{j}^{t}$ number of vehicles at station $j$ at time interval $t$ before other decisions

$\tilde{n}_{j}^{t}$ number of vehicles that should stay at station $j$ at time interval $t$ for safety or charging reasons

$m_{s j}^{t}$ number of personnel of shift $s$ at station $j$ at time interval $t$ before other decisions

$r_{s j}^{t}$ number of vehicles relocated by a personnel from shift $s$ to station $j$ finishing at time interval $t$

$\bar{r}_{s j}^{t}$ number of vehicles relocated by a personnel from shift $s$ from station $j$ starting at time interval $t$

$p_{s j}^{t}$ number of personnel of shift $s$ moved to station $j$ finishing at time interval $t$ without a vehicle

$\bar{p}_{s j}^{t}$ number of personnel of shift $s$ moved from station $j$ starting at time interval $t$ without a vehicle

$\tilde{r}_{s b d}^{t(u)}$ number of vehicles relocated by personnel of shift $s$ from cluster $b$ to cluster $d$ starting at time interval $t$ and finishing at time interval $u$

$\tilde{p}_{s b d}^{t(u)}$ number of personnel of shift $s$ moved from cluster $b$ to cluster $d$ starting at time interval $t$ and finishing at time interval $u$ 


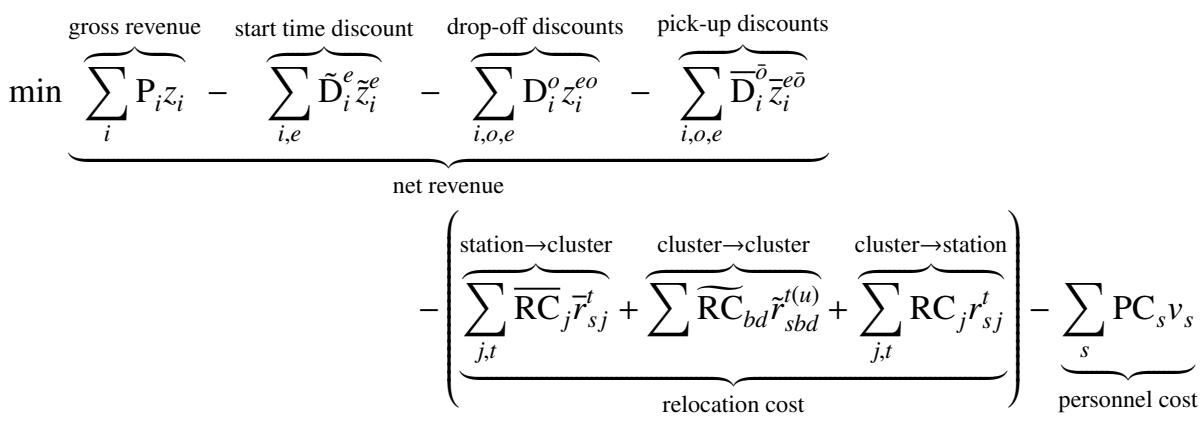

subject to

$$
\begin{aligned}
& \sum_{e \in E_{i}} \tilde{z}_{i}^{e}=z_{i} \quad \sum_{o \in O_{i}^{e}} z_{i}^{e o}=\tilde{z}_{i}^{e} \quad \sum_{o \in \bar{O}_{i}^{e}} \bar{z}_{i}^{e \bar{o}}=\tilde{z}_{i}^{e} \\
& n_{j}^{t+1}=n_{j}^{t}-\sum_{\substack{i, e: \operatorname{start}(i, e)=t \\
\bar{o}: \operatorname{origin}(i, e, \bar{o})=j}} \bar{z}_{i}^{e \bar{o}}-\sum_{s} \bar{r}_{s j}^{t}+\sum_{\begin{array}{c}
i, e: \text { end }(i, e)=t \\
o: \operatorname{dest}(i, e, o)=j
\end{array}} z_{i}^{e o}+\sum_{s} r_{s j}^{t} \\
& n_{j}^{t} \geq \sum_{\substack{i, e: s t a r t(i, e)=t \\
\bar{o}: \operatorname{origin}(i, e, \bar{o})=j}} \bar{z}_{i}^{e \bar{o}}+\sum_{s} \bar{r}_{s j}^{t}+\tilde{n}_{j}^{t} \\
& n_{j}^{t} \leq \mathrm{CAP}_{j}^{t} \\
& 0 \leq n_{j}^{t_{\text {last }}}-\sum_{\begin{array}{c}
i, e: \operatorname{start}(i, e)=t_{\text {last }} \\
o: \text { origin }(i, e, \bar{o})=j
\end{array}} \bar{z}_{i}^{e \bar{o}}-\bar{r}_{s j}^{t_{\text {last }}}+\sum_{\begin{array}{c}
i, e: \text { end }(i, e)=t_{\text {last }} \\
\operatorname{dest}(i, e, o)=j
\end{array}} z_{i}^{e o}+r_{s j}^{t_{\text {last }}} \leq C A P_{j}^{t_{\text {last }}} \\
& \sum_{j} m_{s j}^{\mathrm{start}(s)}=v_{s} \quad \sum_{j} m_{s j}^{\mathrm{end}(s)}=v_{s} \\
& m_{s j}^{t+1}=m_{s j}^{t}-\bar{p}_{s j}^{t}-\bar{r}_{s j}^{t}+p_{s j}^{t}+r_{s j}^{t} \\
& \sum_{d} \tilde{r}_{s b d}^{t(u)}=\sum_{j \in J_{b}} \bar{r}_{s j}^{t-\operatorname{d.from}(j)} \quad \sum_{d} \tilde{r}_{s d b}^{u(t)}=\sum_{j \in J_{b}} r_{s j}^{t+\mathrm{d} \cdot \operatorname{to}(j)} \\
& \sum_{d} \tilde{p}_{s b d}^{t(u)}=\sum_{j \in \bar{J}_{b}} \bar{p}_{s j}^{t-\mathrm{m} \cdot \operatorname{from}(j)} \quad \sum_{d} \tilde{p}_{s d b}^{u(t)}=\sum_{j \in \bar{J}_{b}} p_{s j}^{t+\mathrm{m} \cdot \operatorname{to}(j)}
\end{aligned}
$$

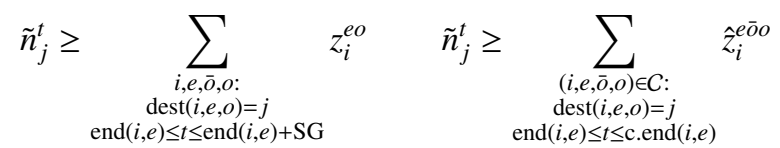

$$
\begin{aligned}
& \hat{z}_{i}^{e \bar{o} o} \geq z_{i}+\tilde{z}_{i}^{e}+\bar{z}_{i}^{e \bar{o}}+z_{i}^{e o}-3 \\
& z_{i}, \tilde{z}_{i}^{e}, z_{i}^{e o}, \bar{z}_{i}^{e \bar{o}}, \hat{z}_{i}^{e \bar{o} o} \in\{0,1\}
\end{aligned}
$$$$
\forall i \text { and } i, e
$$$$
\forall j, t \backslash t_{\text {last }}
$$$$
\forall s, j, t \backslash t_{\text {last }}
$$$$
\forall s, b, t
$$$$
(i, e, o, \bar{o}) \in C
$$$$
\forall i, e, o, \bar{o}
$$$$
\forall s, j, t, b, d
$$

The FOOM is formulated by Eqs. 8-20. The formulation aims to minimize the cost of operating the system. The model inputs are (i) the properties of the system (e.g. driving and moving duration between stations, relocation personnel shifts) and (ii) the cluster sets created by the SCM (see Section 3.1), (iii) demand requests to be served (with alternative start time, origin and destination options). The model output includes the most profitable set of demand (options) to be served and the relocation operations that need to be executed. This model regards each vehicle and personnel as unit flows in separate layers of parallel networks (Fig. 2). The model does not directly check the charging levels of each electric vehicle but eventually becomes charging level feasible with additional constraints proposed by the Joint (Vehicle and Personnel) Flow Model (JFM) (see Section 3.3).

Objective 8 maximizes the profit. We assume that every alternative start time, pick-up or drop-off option offered by the system discount the price of the service. The net revenue earned from a single trip is equal to the gross revenue 
earned from this trip minus the discounts occurring as a result of offering the user start time, and pick-up and dropoff stations different than the user's original choices. The second part of the objective function contains the cost of relocation operations and the personnel hiring. Since the relocation operations are assumed to be done in three separate steps, (1) from station $j$ to the cluster node of $j$, then (2) from the cluster node of $j$ to cluster node of $l$, and finally (3) from the cluster node of $l$ to station $l$, the cost of relocation is also calculated in three separate steps. Personnel cost is calculated by multiplying the unit cost of personnel times the number of personnel working at each shift.

Constraints 9 are used to connect demand $\left(z_{i}\right)$ and option $\left(\tilde{z}_{i}^{e}, \bar{z}_{i}^{e \bar{o}}, z_{i}^{e o}\right)$ variables. Constraints 9 a ensure that if demand $i$ is served, only one of the start time option (e) is selected. If demand $i$ is not served, then none of the start time option is served. We use similar approaches for the other two constraints. Constraints $9 \mathrm{~b}$ and c ensure that if one of the start time options is served $\left(\tilde{z}_{i}^{e}\right)$ one of the origin $\left(\bar{z}_{i}^{e \bar{o}}\right)$ and destination $\left(z_{i}^{e o}\right)$ options are served.

Constraints 10 ensure vehicle flow conservation is satisfied. The number of vehicles at station $j$ at the beginning of time interval $t+1$ is equal to the number of vehicles at station $j$ at the beginning of time interval $t$ plus the net number of vehicles arriving to the station at time interval $t$. Net number of vehicles arriving to the station is equal to the number of vehicles arriving from rentals or relocation operations minus the number of vehicles departing for rentals or relocation operations from station $j$ at time interval $t$. The flow diagram located on the left of Fig. 3 illustrates this relationship.

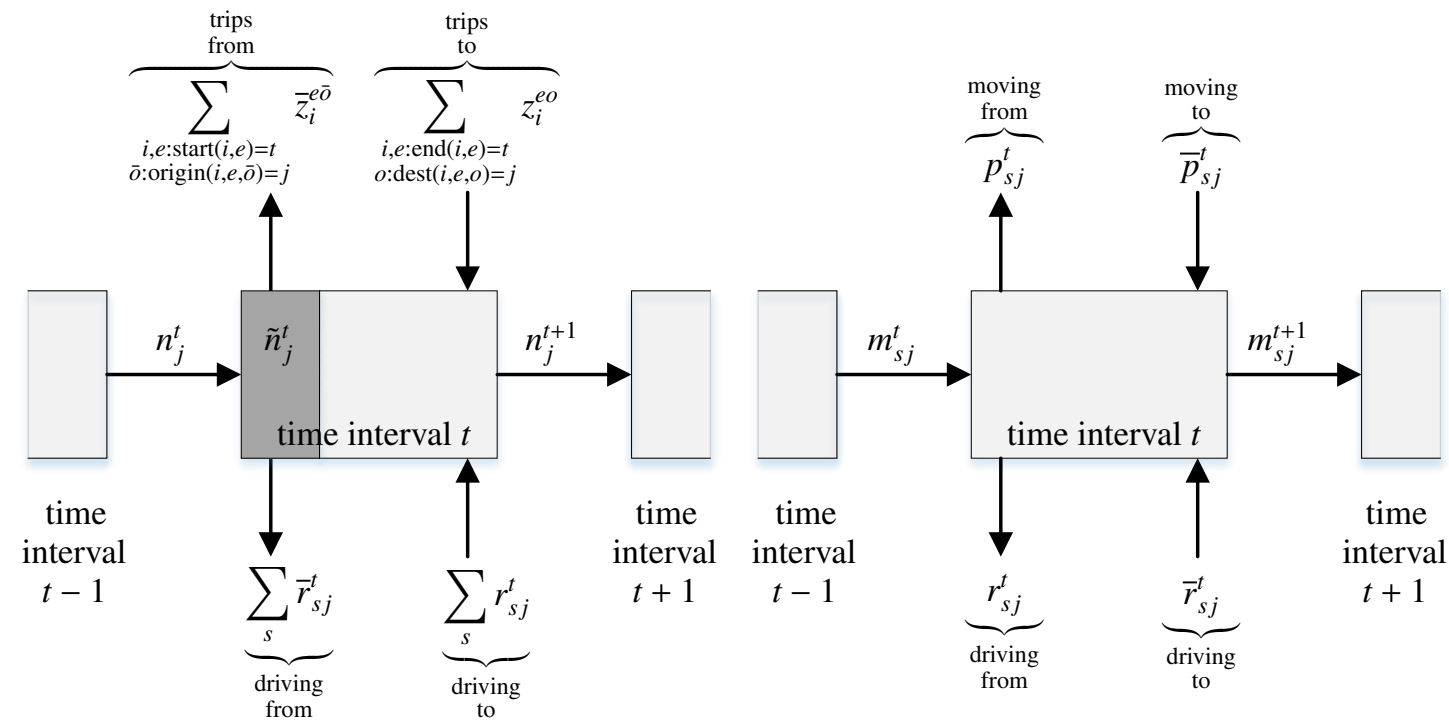

Figure 3: Flow diagram of vehicles (left) and personnel shift $s$ (right) in station $j$ at time interval $t$.

Constraints 11a require that the number of vehicles at station $j$ at the beginning of time interval $t$ is more than the sum of the number of demand $\left(\bar{z}_{a_{i}}^{a_{i}, a_{o}}\right)$ and driving operations $\left(\bar{r}_{s j}^{t}\right)$ destined from this station, and the number of vehicles forced to be charged at this station during time interval $t$. With these constraints we ensure that any order of driving operations and rentals departing from this station is feasible. Constraints $11 \mathrm{~b}$ restrict the number of vehicles at the station with the capacity of the station.

Constraints 12 are introduced to the model to restrict the number of vehicles at each station at the end of last time intervals. Number of vehicles at the beginning of the last time interval plus the number of vehicles arriving to the station from rentals or relocation operations minus the number of vehicles leaving the station for rentals or relocation operations should be between 0 and the capacity of the station.

Constraints 13 are used to keep track of the number of personnel assigned to each shift. Constraints $13 \mathrm{a}$ and $\mathrm{b}$ require that the sum of the number of personnel from shift $s\left(m_{s j}^{t}\right)$ at all stations at the beginning and end of time intervals of the same shift should be equal to the number of personnel assigned $\left(v_{s}\right)$ to this shift.

Constraints 14 are flow conservation equations for personnel. Number of personnel at station $\mathrm{j}$ at the beginning of time interval $t+1$ from shift $s$ is equal to the number of personnel at station $j$ at the beginning of time interval $t$ 
plus the net number of personnel arriving to the station from shift $s$. The net number of personnel arriving to station is equal to the the number of personnel arriving to the station with $\left(r_{s j}^{t}\right)$ or without $\left(p_{s j}^{t}\right)$ vehicles minus the number of personnel departing from the station with $\left(\bar{r}_{s j}^{t}\right)$ or without vehicles $\left(\bar{p}_{s j}^{t}\right)$. The flow diagram located on the right of Fig. 3 illustrates this relationship.

Constraints 15 and 16 are required to have feasible relocation arcs that form feasible relocation operations when they are joined. They are simply flow conservation equations of relocation flows for the cluster nodes. Constraints $15 \mathrm{a}$ and $16 \mathrm{a}$ require that the number of relocation flows with and without vehicle originating from the cluster node $b$ at time interval $t$ should be equal to the sum of the relocation flows arriving to cluster node $b$ at time interval $t$ from the nodes of cluster $b$. Similarly, constraints $15 \mathrm{~b}$ and $16 \mathrm{~b}$ require that the number of relocation flows with and without vehicle destined to $b$ at time interval $t$ should be equal to the sum of the relocation flows departing from cluster node $b$ at time interval $t$ to the nodes of cluster $b$.

Constraints 17 are required to force vehicles to stay at the stations for either safety or charging purposes. Constraints 17 a consider that the rented vehicles could be returned to their destination station later than the reserved end time. To ensure the quality of service, we do not allow users to rent these returned vehicles for a while. We keep a spot with the returned vehicle at the destination station starting from the end time interval of the demand for safety gap (SG) many time intervals. With these constraints, even if the vehicle is returned later than the reservation end time, as long as it is within safety gap limits, it does not leave any accepted rentals without vehicles. Constraints $17 \mathrm{~b}$ ensure that if any trips are in the forced charging set $C$, the vehicle that is serving one of these exact trips should be kept for a while at the station until it is charged enough to cover the energy consumption of the trip. Note that, constraints 18 force the variable $\hat{z}_{i}^{e \bar{o} o}$ to be 1 only if exactly the same demand is served with the same options. In other words, if the demand $i$ is not served with start time option $e$, origin option $\bar{o}$ or destination option $o$ which was forced to be charged in one of the previous iterations, then any vehicles will not be forced to stay and charged at the station after serving the trip.

Constraints 19 and 20 define the domains of each variables. Variables related to demand and their options are set to be binary. The rest of the variables that keep track of the number of vehicles and personnel at each node, and the number of relocation operations executed are all set to be non-negative integers.

Boyac1 et al. (2017) propose a similar operations optimization model, constraints similar to 10-17 exist in the referred work. However, the implementation of spatial and temporal flexibility, and using station clustering model that supports asymmetric travel times require changes in the modelling approach. Different than the model proposed by Boyac1 et al. (2017), in constraints 10-12, instead of demand, we use origin and destination options of the demand requests. Similarly, in constraints 15 and 16, the superscripts of $r_{s j}^{t}\left(\bar{r}_{s j}^{t}\right)$ and $p_{s j}^{t}\left(\bar{p}_{s j}^{t}\right)$ are changing according to the travel times from (to) these nodes to (from) their cluster nodes. There are also additional unique constraints in this proposed model (e.g. 9, 18) that are not proposed by Boyac1 et al. (2017).

\subsection{Joint (Personnel and Vehicle) Flow Model (JFM)}

The Joint (Personnel and Vehicle) Flow Model (JFM) considers both the movements of personnel and vehicles, and charging levels of the vehicles at the same time for all shifts together. Although it is possible and easier to solve personnel and vehicle flow problems separately, dealing with both problems within a single model provides better results in finding charging level feasible solutions. For given vehicle and personnel flows, taking both the personnel assignment and vehicle flow decisions at the same time with the JFM explores the entire solution space whereas solving each problem sequentially discards part of the solution space.

\subsubsection{Sets and indices}

$$
\begin{aligned}
& k \in K \text { (individual) vehicles } \\
& q \in Q \text { (individual) personnel } \\
& s \in S \text { working shifts } \\
& j \in J \text { stations } \\
& t \in T \text { time intervals } \\
& b, d \in B \text { clusters } \\
& f \in F \text { (relocation and rental) flows }
\end{aligned}
$$




\subsubsection{Parameters}

$n_{j}^{t} / m_{s j}^{t}$ number of vehicles / personnel at station $j$ at the beginning of time interval $t$

$r_{s j}^{t} / p_{s j}^{t}$ number of personnel finishing driving / moving to station $j$ at time interval $t$

$\bar{r}_{s j}^{t} / \bar{p}_{s j}^{t}$ number of personnel starting driving / moving from station $j$ at time interval $t$

$\tilde{r}_{s b d}^{t(u)} / \tilde{p}_{s b d}^{t(u)}$ number of personnel driving / moving from cluster node $b$ to $d$ starting at time interval $t$

$v_{s}$ number of personnel from shift $s$

$\operatorname{origin}(f) / \operatorname{dest}(f)$ origin/destination node of flow $f$

$\operatorname{start}(f) /$ end $(f)$ start / end time interval of flow $f$

c.end $(f)$ charging end time interval of flow $f$

$\operatorname{dur}(f) / \operatorname{dis}(f)$ duration / distance of flow $f$

$\operatorname{shift}(f)$ shift of relocation flow $f$

type $(f)$ type of flow $f$ (D: driving, M: moving, S: rental, R: relocation $=\mathrm{D} \cup \mathrm{M}, \mathrm{V}$ : vehicle $=\mathrm{D} \cup \mathrm{S}$ )

$J_{b} / \bar{J}_{b}$ set of stations of driving / moving cluster $b$

d.from $(j) / \mathrm{m}$.from $(j)$ number of time intervals needed to drive/move from station $j$ to its cluster node

d.to $(j) /$ m.to( $j)$ number of time intervals needed to drive/move from cluster node of station $j$ to $j$

$\operatorname{MC}(f)$ minimum charging level required to serve flow $f$

$\Delta \mathrm{C}(f)$ percent of charging level used to serve flow $f$

$\mathrm{CR}(j)$ charging rate of station $j$ (percent charged per time interval)

$C$ set of flows that need charging

\subsubsection{Variables}

$\tilde{x}_{j k}^{t} / x_{s j q}^{t} 1$ if $\{$ vehicle $k\} /\{$ personnel $q\}$ is at station $j$ before any decisions at time interval $t ; 0 \mathrm{o} / \mathrm{w}$

$\tilde{y}_{k f} / y_{q f} 1$ if flow $f$ is served by \{vehicle $\left.k\right\} /$ personnel $\left.q\right\} ; 0 \mathrm{o} / \mathrm{w}$

$c_{k}^{t}$ charging level of vehicle $k$ before any decisions at time interval $t$

$\tilde{c}_{k f} 1$ if flow $f$ served by vehicle $k$ is assumed to spend no charge at all (infeasible charging); $0 \mathrm{o} / \mathrm{w}$ 
$\min \sum_{k, f} \tilde{c}_{k f}$

subject to

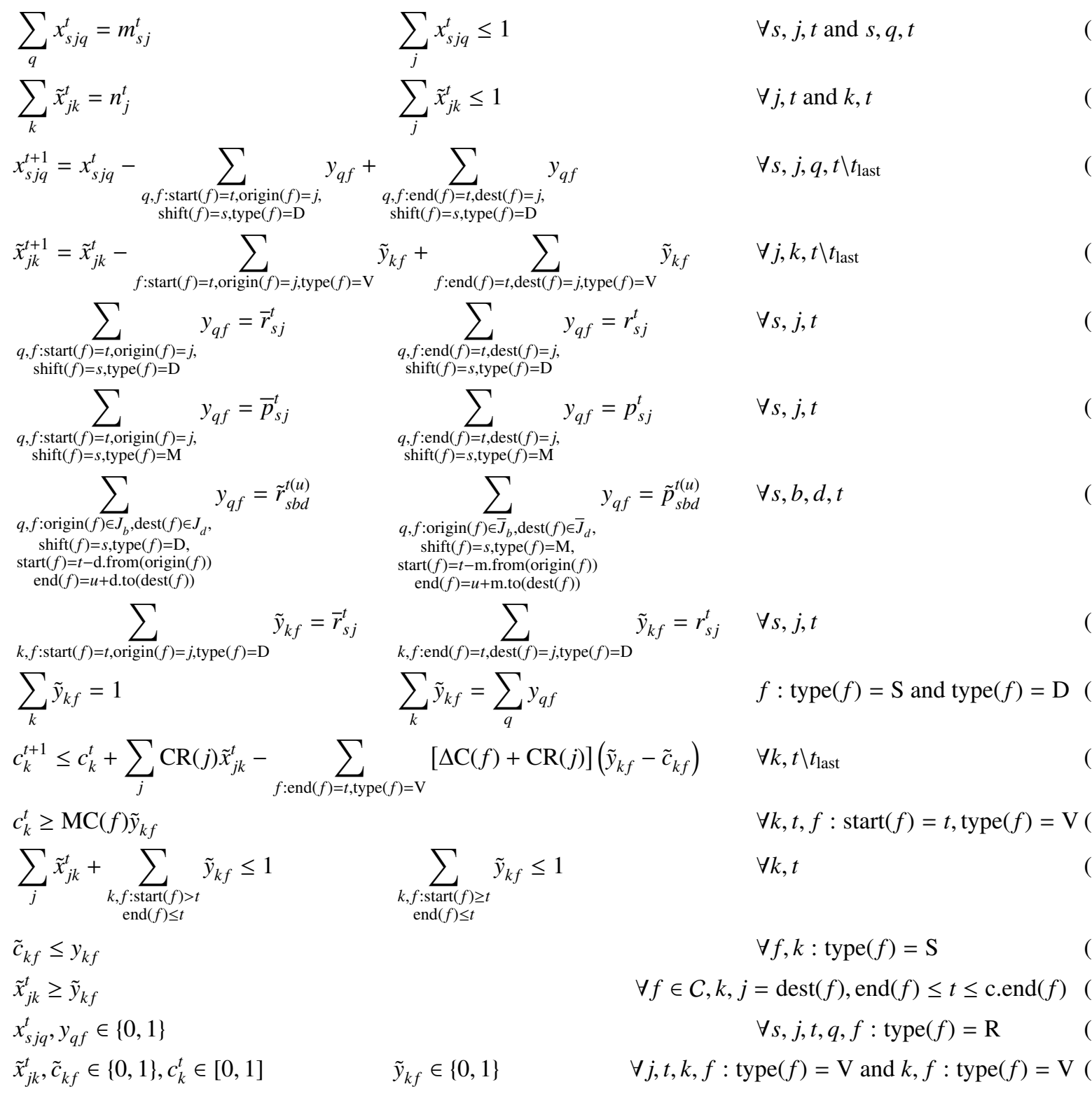

The Joint (Personnel and Vehicle) Flow Model (JFM) converts unit personnel and vehicle flows into feasible assignments for each individual personnel and vehicles. Note that, any solution created by the FOOM corresponds to at least one feasible solution that assigns each personnel and vehicle to unique flows. However, these solutions are not necessarily charging level feasible for the vehicles. That is the reason, in this model, our aim is to minimize the number of infeasible charging levels occurring in the objective with Eq. 21.

Constraints 22a and 23a postulate that the number of personnel and vehicles at each node $j$ during each time interval $t$ stated by the FOOM should be equal to the number of individual personnel and vehicles assigned to the same nodes respectively. Note that constraints 22 also iterates over the shifts since the FOOM differentiates personnel 
according to their shifts. Constraints $22 \mathrm{~b}$ and $23 \mathrm{~b}$ ensure that any individual personnel and vehicles cannot be in more than one stations during the same time interval respectively.

Constraints 24 and 25 are flow conservation equations for individual personnel and vehicles respectively. These constraints ensure that if personnel $q$ from shift $s$ (vehicle $k$ ) is at station $j$ at time interval $t+1$, either the personnel (vehicle) was there during time interval $t$ and not depart for any relocation (and trip) starting at time $t$ or has arrived from another relocation (and trip) ending at time interval $t$.

Constraints 26, 27 and 28 ensure that every relocation flow is covered by one and only one personnel. As stated before, relocation operations are modelled with three separate flows instead of one in the Flexible Operations Optimization Model: A relocation with (without) vehicle from station $j$ to $l$ is modelled as (1) from station $j$ to its cluster node $B_{j}\left(\bar{B}_{j}\right),(2)$ from cluster node $B_{j}\left(\bar{B}_{j}\right)$ to cluster node of station $l, B_{l}\left(\bar{B}_{l}\right)$ and (3) from cluster node $B_{l}\left(\bar{B}_{l}\right)$ to station $l$. Constraints $26 \mathrm{a}(26 \mathrm{~b})$ deal with the first (third) step of these flows and postulate that the number of relocation operations with vehicles from (to) station $j$ starting (ending) at time interval $t$ executed by a personnel from shift $s$ stated by the FOOM should be equal to the total number of individual personnel from shift $s$ assigned to exactly the same number of relocation operations with vehicles starting (ending) at time interval $t$ from (to) station $j$. Constraints $27 \mathrm{a}(27 \mathrm{~b})$ postulate exactly the same restrictions for the relocation operations without vehicles for the personnel flows. Constraints $28 \mathrm{a}$ and $28 \mathrm{~b}$ deal with the second step of relocations with and without vehicles respectively. Constraints $28 \mathrm{a}(28 \mathrm{~b})$ postulate the number of relocation operations with (without) vehicles from stations that belong to cluster $b$ to stations that belong to cluster $d$, reaching to cluster $b$ at time interval $t$ should be equal to the number of individual relocation personnel assigned to these operations.

Constraints 29a (29b) ensure that the number of relocation operations with vehicles starting (ending) at time interval $t$ from (to) station $j$ stated by the FOOM are equal to the total number of separate vehicles assigned to the flows starting (ending) at time interval $t$ from (to) station $j$.

Constraints 30a postulate that each accepted rental should be assigned to one and only one vehicle flows. Similarly, constraints $30 \mathrm{~b}$ ensure that the total number of relocation flows with vehicles assigned to any individual personnel should be equal to the total number of separate vehicle flows assigned to the same flows. Note that constraints $30 \mathrm{a}$ and $30 \mathrm{~b}$ hold for every flow from the set of trips (S) and relocation operations with vehicles (D) respectively.

Constraints 31 are used to keep track of the charging level of each vehicle. The charging level of a vehicle at the beginning of time interval $t+1$ should be greater than or equal to the charging level of the same vehicle at the beginning time interval $t$, plus the charging rate of the station that the vehicle is being charged (if it is parked at a station), minus the charging level spend for the latest flow the vehicle is serving. The constraints are holding with inequality rather than equality since the vehicle battery cannot be charged more than its capacity. Note that, we subtract the charging rate if a flow is served since variable $\tilde{x}_{j k}^{t}$ shows the state of the vehicle before any decisions including flows. If a vehicle is serving a flow, it cannot be charged during the period it starts serving the flow. We also subtract $\tilde{c}_{k f}$ from $\tilde{y}_{k f}$. If a flow cannot be served because of the charging level at the end of the flow, $\tilde{c}_{k f}$ gets value of 1 which shows us we need to force the vehicle to charge its batteries that serves flow $f$ after serving in the next FOOM. Last but not least, although in reality the charging levels of the vehicles drop continuously, we deduce the charging level of the vehicles at the end of the flows. Since we are comparing the charging levels at the beginning of the flows with the minimum charging levels needed to serve the flows, dropping charging levels at the end of the flows is an acceptable assumption.

Constraints 32 ensure that the vehicle serving flow $f$ has enough energy in its battery. As stated before, the charging level of the vehicles are checked only at the very beginning of the flows.

Constraints $33 \mathrm{a}$ and $33 \mathrm{~b}$ are redundant cuts added to the model to decrease the solution space and time eventually. Constraints 33 a state that the same vehicle cannot be in two places at the same time. It cannot stay at different nodes while serving different flows happening at the same time. If flow $f$ is served by vehicle $k$, at the first time interval of flow $f$ vehicle is at the origin node of flow $f$. That is the reason, in constraints 33a, the second summation starts from the time interval after the start time interval of the flows. Constraints $33 \mathrm{~b}$ state that every vehicle can serve at most one flow at each time interval.

As stated before, $\tilde{c}_{k f}$ gets value of 1 if at the beginning of flow $f$, the charging level of vehicle $k$ is not enough to serve the same flow. With constraints 34 , we set the relationship between variables $\tilde{y}_{k f}$ and $\tilde{c}_{k f}$ for the same flow $f$ and vehicle $k$. If vehicle $k$ is not serving flow $f$ (i.e. $\tilde{y}_{k f}=0$ ), then the variable $\tilde{c}_{k f}$, which controls if the vehicle $k$ serve flow $f$ without enough charging level, cannot have value of 1 .

At every iteration, the FOOM keeps track of flows that require forced charging after they are being served. With 
constraints 35 , we satisfy these restrictions. If the flow is in the forced charging set $(C)$ then the vehicle serving flow $f$ is forced to stay at the destination station and charge itself after serving $f$. If the vehicle is in $C$, it is required to stay at the destination station from flow end time end $(f)$ to charging end time c.end $(f)$.

Constraints 36 and 37 set the domains for the variables used for the individual personnel and vehicles respectively. All the variables except $\tilde{c}_{k f}$ are binary variables. $\tilde{c}_{k f}$ represents the percent charging level of vehicles at each time interval and can take any values between 0 and 1 .

\subsection{Personnel Flow Model}

Although the JFM always gives the assignment set that minimizes the number of trips that are served by vehicles with infeasible charging levels, solving the problem by using the JFM is more time consuming than solving personnel and vehicle flow problems separately. In this section, we will describe the Personnel Flow Model (PFM) that creates assignments for personnel only and do not consider vehicles and their charging levels at all.

The PFM is similar to the model formulated by Boyac1 et al. (2017). However, since in the clustering model we are considering a travel time between nodes and their clusters, to reflect this requirement, appropriate changes has been made in the new formulation. The personnel flow problem can be modelled as follows by using the personnel flow related indices, parameters and variables defined in the JFM in Section 3.3.

$$
\begin{aligned}
& \min \sum_{s, f, k} \operatorname{dis}(f) y_{q f} \\
& \text { subject to (22), (24), (26), (27), (28), (36). }
\end{aligned}
$$

Since the PFM is not considering vehicles and their charging levels, we use the distances of complete relocation flows formed. Although this approach does not necessarily increase the chance of having charging level feasible assignments for the vehicles in the next stage of the solution, minimizing the distance travelled in relocation operations consumes less energy and eventually keeps on requirements that the sum of the charging levels of vehicles are higher.

Note that, this model can also be solved separately for each shift $s$ without the loss of generality since each relocation operation and as a result the flows generated from them are assigned to the shift executing them.

\subsection{Vehicle Assignment Simulation}

The Vehicle Assignment Simulation (VAS) is the fastest but least accurate approach in searching charging level feasible solutions. It is fast because it is a discrete event simulation which uses a simple policy in assigning vehicles to trip or relocation requests. When there are more than one vehicles which are located at the origin station of a trip or driving request, the simulation assigns the vehicle with the highest charging level. Assignment policy orders the requests in decreasing order of charging level need and the vehicles in decreasing order of charging levels. Subsequently the requests and vehicles are assigned to each other in the same order. This myopic approach returns a charging level feasible solution in most of the cases. However, it is not exploring the entire solution space, therefore it may miss a feasible solution especially in cases at which vehicles are more intensively utilized.

The algorithm works as follows. After solving the Personnel Flow Model, we end up with the exact number of flows that the system should serve between stations at each time interval. We create two events by using the start and end times of each flow and add them to the event list. The event list orders events by using two criteria. Initially it ranks the events in terms of increasing time. In the case that multiple events are in the same ranking of the event list, the charging needs of the requests are used to produce the final order of the events. This means that the events with higher charging needs are served first. Pick-up events happen to be ordered first in case of a tie. Next, we generate the vehicles and initialize the locations of their first stations. We also keep vehicle lists for each station and keep vehicles in decreasing order of charging levels. Then we iterate over the items of the event list. First we update the charging levels of the vehicles located at the stations by calculating the time difference between the current and previous event. We then assign the vehicles to pick-up events originating from the stations that the vehicles are located. We check if the current charging level of the vehicle is more than the minimum charging level required by the flow of the event. If the condition is not satisfied, the algorithm stops and provides an infeasible solution. If the condition is satisfied, the algorithm deduces the charging level needed to serve the trip by this vehicle and removes the vehicle from the station list. If the item is a drop-off event, then the vehicle assigned for the pick-up event of this flow is assigned to the 
destination station of the same event. If all the items in the event list are executed without any problems, the algorithm stops and returns a feasible solution.

This algorithm is very similar to the simulation module described in Boyac1 et al. (2017). However, in order to increase the efficiency of the algorithm, we have removed the personnel objects and activities from the simulation, and regard trips and relocations of vehicles as identical activities with different charging level requirements.

\section{Model application}

We applied the solution framework represented in Fig. 1. We use the data gathered from the one-way electric carsharing system of Auto Bleue operating in Nice, France (Auto Bleue, 2018). Auto Bleue is operating a oneway system since 2014 . We use the anonymized one-way rental and publicly available station location data. In our analyses, we considered only 60 stations located in the city center and excluded the 6 stations in the suburbs (Fig. 4). This was dictated by the fact that these 6 stations were rarely used or not allowed to be used for one-way trips in the data. In order to stress the system resource usage with higher demand levels, we aggregated data from individual days, and added spatial and temporal flexibility for different options in our runs.

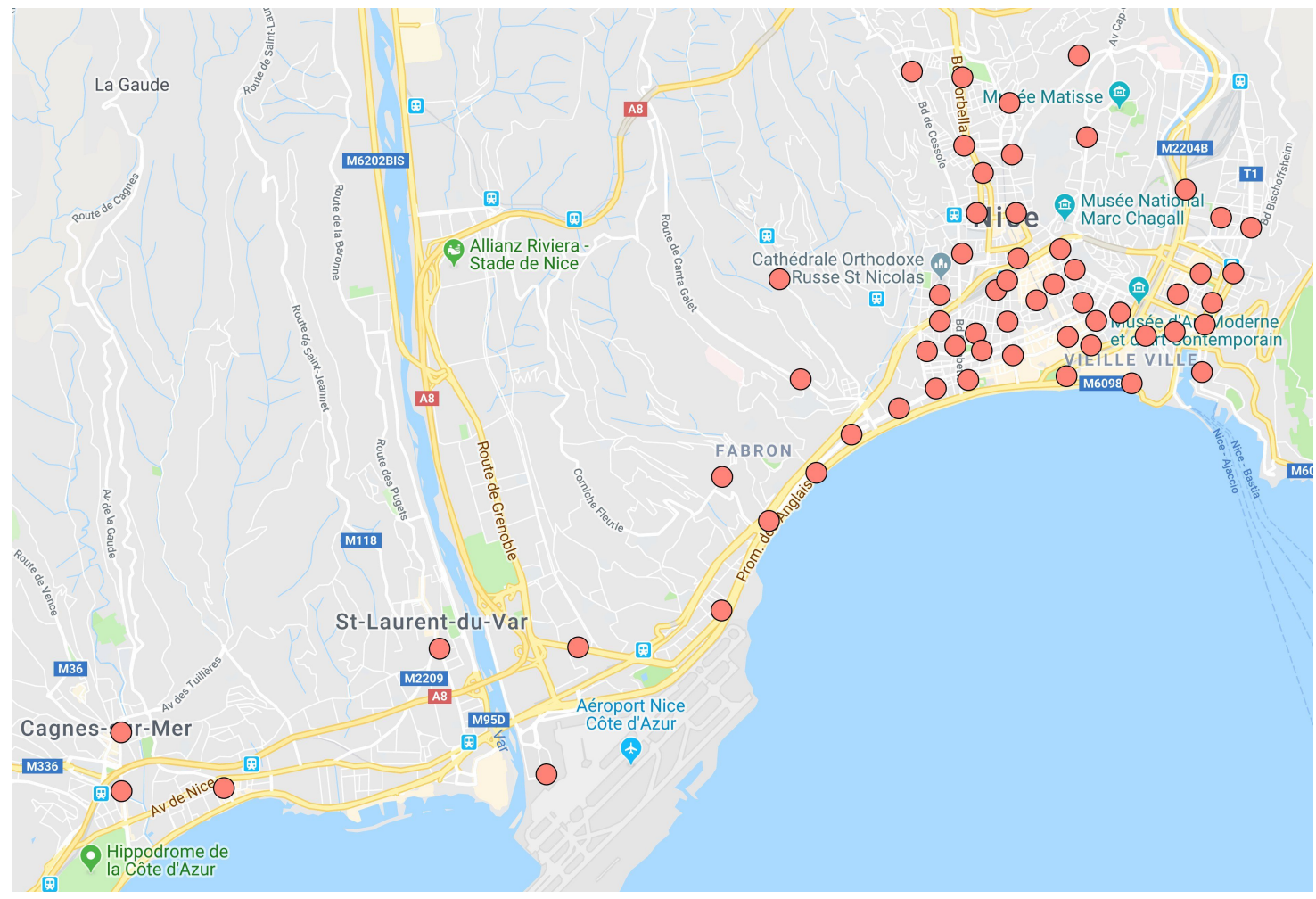

Figure 4: Locations of 60 stations considered in the analyses.

We implemented the model with C\# .NET environment. IBM ILOG Cplex 12.8 is used to solve MILPs. Experiments are conducted on a workstation with Intel Xeon E5-2640 v3 processor.

The proposed solution framework assumes the states of the system components change in discrete times, i.e. time intervals. In our experiments, we set the duration of each time intervals to 15 minutes. A vehicle or a personnel can have only one state during a time interval. The relocation personnel is assumed to either drive a car (driving) or cycle a bike (moving) between stations. We use the driving and cycling distances that are provided by Google Maps API (Google, 2018) and assumed driving and moving speeds are 30 and $15 \mathrm{~km} / \mathrm{h}$ respectively. Please note that, although the same model could be used for different relocation speeds throughout the day for any station pairs, we keep the same speed for the simplicity of our analyses. We use 4 different personnel shifts: 6:00-09:45, 09:45-13:45, 13:45-17:45 and 17:45-21:45. The other important parameters we used in our analyses are given in Table 2. 
Table 2: Important parameter values used in the analyses.

\begin{tabular}{|l|l|}
\hline relocation personnel cost $(€ /$ shift): & 72 \\
relocation (fuel) cost $(€ / \mathrm{km}):$ & 0.02 \\
charging duration per distance $(\mathrm{sec} / \mathrm{km}):$ & 240 \\
vehicle range $(\mathrm{km}):$ & 120 \\
minimum trip start charging level & $40 \%$ \\
min-max (mean) trip distance $(\mathrm{km}):$ & $3-40(18)$ \\
min-max (mean) trip duration (hour): & $.2-10(.8)$ \\
rental fee (€/hour): & 18 \\
spatial flexibility discount $(€ / \mathrm{km}):$ & 4 \\
temporal flexibility discount $(€ /$ hour): & 9 \\
minimum time between pick-up and drop-offs (min): & 30 \\
\hline
\end{tabular}

\subsection{Station clustering}

We started our analyses with the station clustering for both driving and moving operations. Since there are 60 stations and 64 time intervals (from 6:00 to 22:00 with 15 minute intervals), without clustering, we need to deal with over $450000(2 \times 60 \times 59 \times 64)$ relocation variables. Using station clustering (see Section 3.1$)$, the relocation operations are modelled through cluster nodes, therefore the number of relocation arcs and eventually variables is decreased and the model can be solved for real world instances.

In clustering, we use the mathematical model represented in Section 3.1. We solve the mathematical model for different number of clusters and relocation operations. We generate different clusters for driving and moving operations separately since the relocation time between each pair of stations differs according to the type of relocation operations.

In order to decrease the size of the clustering model, we preprocess the problem and identify the stations that will take the same number of time intervals to travel to all other stations. In other words, we identify stations that should be at the same cluster in an optimal solution. In the mathematical model, we regard these station groups as the same stations. This approach decreases the problem size for driving from 60 stations to 29 station groups. Unfortunately the same approach does not change the problem size for moving operations.

When we apply the clustering model, we use objective function weights (see Eq. 1) proportional to the number of relocations happening between station pairs. This approach minimizes the total error emerging due to clustering. However, since the relocation operations are decisions generated by the Flexible Operations Optimization Model, it is not possible to find how many relocations are happening between every station pairs. In order to define this number, we assume that the number of relocations happening between stations (or station groups) should be directly proportional to the demand originating from and destined to these two stations (or station groups). We normalize the weights by dividing all original weights to the sum of the number of relocation operations.

After applying the preprocessing and setting the solution time to 24 hours, we run the algorithm for different number of clusters. The weighted error emerging from the clustering approach for using different number of clusters is given in Fig. 5.

As it is stated above, we are using the clustering approach to have more efficient solution framework while keeping the accuracy within an acceptable range. Using too many clusters reduces the overestimation error associated with the duration of relocation operations results to a more accurate model albeit with more demanding computational difficulties. In contrast, the use of too few clusters leads to a higher overestimation of the duration of relocations but to an easier problem to solve. After analyzing results given in Fig. 5 we decided that 13 clusters for driving and 15 clusters for moving provide us acceptable efficiency and accuracy levels for our framework. After these decisions, the clusters are formed as illustrated in Fig. 6. The left and right maps present 13 and 15 clusters used for driving and moving operations respectively. Beyond these points, there are diminishing rate of improvements of the solutions. The matrices located on the top left of the maps state the number of intervals to travel from the cluster nodes representing stations on the first column to the cluster nodes representing stations on the first row. Most of the travel from/to cluster nodes to/from stations happen instantaneously. There are only a few nodes (two in driving and eight in moving cases) from/to which it takes positive number of time intervals to travel. These nodes are shown on the figure with numbers 

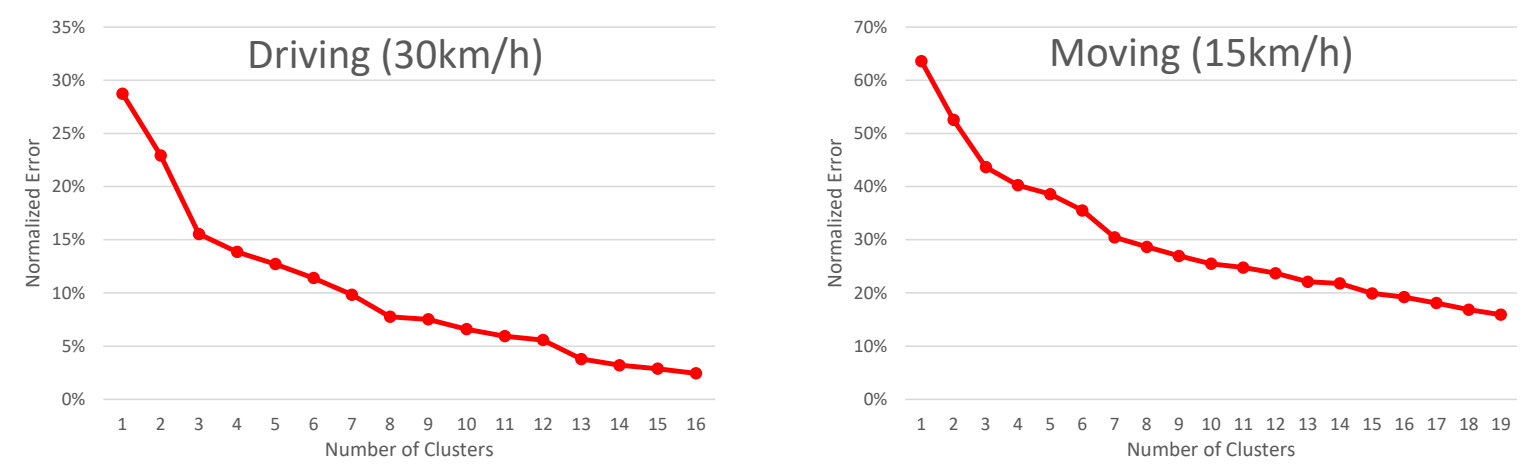

Figure 5: Normalized error emerging from using different number of clusters for driving (left) and moving (right) operations.

on the upper-left of the nodes. The first number shows the number of time intervals required to travel from this node to the cluster node. Similarly, the second number shows the number of time intervals required to travel to this node from its cluster node. These two variables are represented with $u_{j}$ and $\bar{u}_{j}$ in the Station Clustering Model (see Section 3.1) respectively.
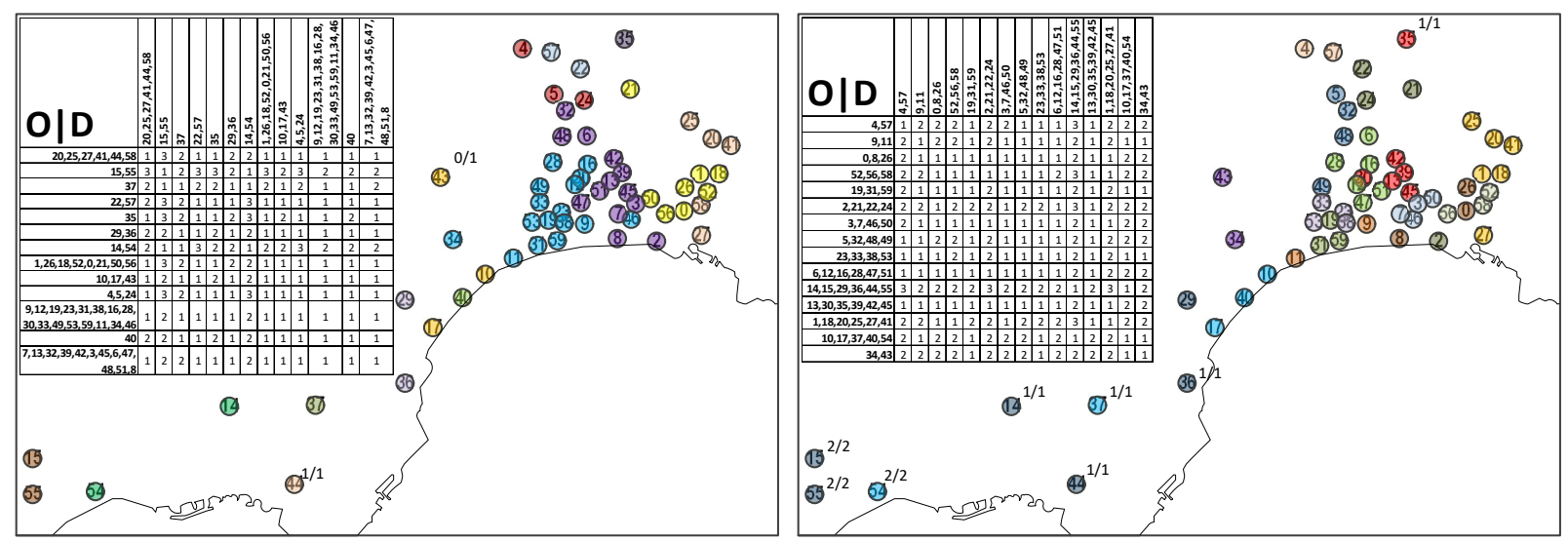

Figure 6: Clusters for driving (left) and moving (right) generated by the Station Clustering Model (see Sec. 3.1). The matrices on the left top shows the number of time intervals needed to travel from cluster node of stations on the first column to the cluster node of station on the first row. If the travel time from/to any node to/from its cluster is different than zero, these nodes contain a value pair on top of their representations. The number pairs show the number of time intervals needed to travel from/to this node to/from its cluster node.

In the subsequent sections, we demonstrate the capabilities of the proposed framework to analyze:

- The effect of spatial and temporal flexibility

- The effect of service type

- The decisions and performance of an instance

We would like to iterate that these findings are relevant to system under consideration and the incentive values assumed. The objective of these analyses is to demonstrate the capabilities of the proposed framework, to investigate the introduction of temporal and spatial flexibility into one-way electric carsharing systems, and service types. It is clear that the acceptability of the alternative trip offers to those originally requested by the users and the associated incentives depends entirely on the user behaviour. User behaviour modelling has not been introduce to this paper. However, the outcomes of a user behaviour study in terms of the acceptability of alternative trips and incentives can be used as an input to our model. Instead of the assumed values used in this paper, values reflecting the mutual behaviour of the users (as defined by user acceptability models) can be used in our model. 


\subsection{The effect of spatial and temporal flexibility}

We first analyze the effects of the spatial and temporal flexibility level offered to the users. We assumed in these experiments that the users are offered alternative trips originating from and destined to different stations, starting at different times than originally requested by the users. We limit the alternative offers to the users to be within a predefined range of the original request's origin and destination stations, and pick-up times. Travel times and driving distances requested by the users are assumed to stay the same. If a user starts a trip earlier or later than the original trip, he/she finishes the trip earlier or later with the same offset. In these instances, we assume the decision on demand is done with perfect information: The entire set of demand requests are known to the solution framework in the process of decision (see Service Type 1 in Section 4.3).

We use 5 different temporal flexibility levels (i.e. $0,15,30,45$ and 60 minutes earlier or later) for the time between original and offered pick-up times. A discount is applied to the price of the service if the offered trip that is different than the original request is chosen to be served by the operator. Serving the trip not at the time of original request decreases the revenue earned from this trip. Similarly, five different spatial flexibility levels (i.e. $0,0.25,0.5,0.75$ and $1 \mathrm{~km}$ ) are used for the distance between the requested and offered origin and destination stations. We limited the distance flexibility to be half of the original trip length: A station more than half of the original travel distance away from the original origin and destination stations are not offered as alternative stations. We conduct the experiments for 3 different demand levels (300, 400 and 500 requests/day) and repeated the experiments with 10 different demand sets.

Fig. 7 demonstrates the 3 important service measures of one-way systems: demand served (first row), profit earned (second row) and personnel count (third row). We create these figures from 10 different instances by running with different flexibility levels. For every instance, we take the runs with no flexibility option as the base case for every instance and depict the average improvements from these base cases to show percent improvements.

For the system under consideration, we observe for the same level of demand, the spatial flexibility has significant effect on the percentage increase in the number of trips served (top row in Fig. 7). In the base cases of 300, 400 and 500 demand instances, the systems have served on average 202.6, 248.1 and 276.5 customers respectively. With the increase in spatial flexibility, we see 20-30\% increase in the number of demand served. This effect is apparent when the spatial flexibility is beyond 250 meters. This is because the system has only 3 station pairs that are within 250 meters distance. Temporal flexibility has also an effect but not as strong as the spatial flexibility. In combination with spatial flexibility increase, the increase in demand has also positive effect on the percentage of trips served in addition to the base case, i.e. instances with no flexibility. In other words, as the number of demand request increases in the system, the percent increase gained with spatial flexibility also increases. Instances with 300 demand increase the number of demand served by $20 \%$, whereas the instances with 400 and 500 demand increase the number of demand served by 25 and $30 \%$ respectively.

The profitability of the system (middle row in Fig. 7) also increases with the increase in spatial flexibility. Compared to base case with no flexibility, we observe over $10 \%$ improvement in the revenue when the spatial flexibility is increased to 1000 meters. Temporal flexibility has also a slight effect but it is nowhere near to the effect of the spatial flexibility. In combination with spatial flexibility increase, the increase in demand increases the profits slightly. This effect is not as strong as the one we see in the percentage increase in the number of trips served but it is apparent that the instances with more demand gains proportionally more. The instances with 500 demand increase their profit over $10 \%$ on average with maximum flexibility whereas the instances with 300 and 400 demand have a profit increase just under $10 \%$.

We also analyze the number of personnel (bottom row in Fig. 7 since it is an additional challenge for one-way systems that the operators do not want to deal with. In those figures, we depict the percent decrease of personnel cost from the base instance with no flexibility. Since we are using standard personnel shifts in our instances, the personnel cost is directly proportional to the number of personnel in the system. In the base instances of 300, 400 and 500 demand requests, on average there are 4.5, 6.1 and 7.6 personnel respectively. In both 3 demand levels, we have observed that the spatial flexibility of 1000 meters decreases the personnel costs by almost $80 \%$. Again the temporal flexibility has a slight effect but it is not as strong as the spatial flexibility.

\subsection{The effect of service type}

In the second set of analyses we look for the effect of the 3 different service type configurations compared to base configuration. In Service Type 1, we assume that the solution framework processes the entire demand all at once and 


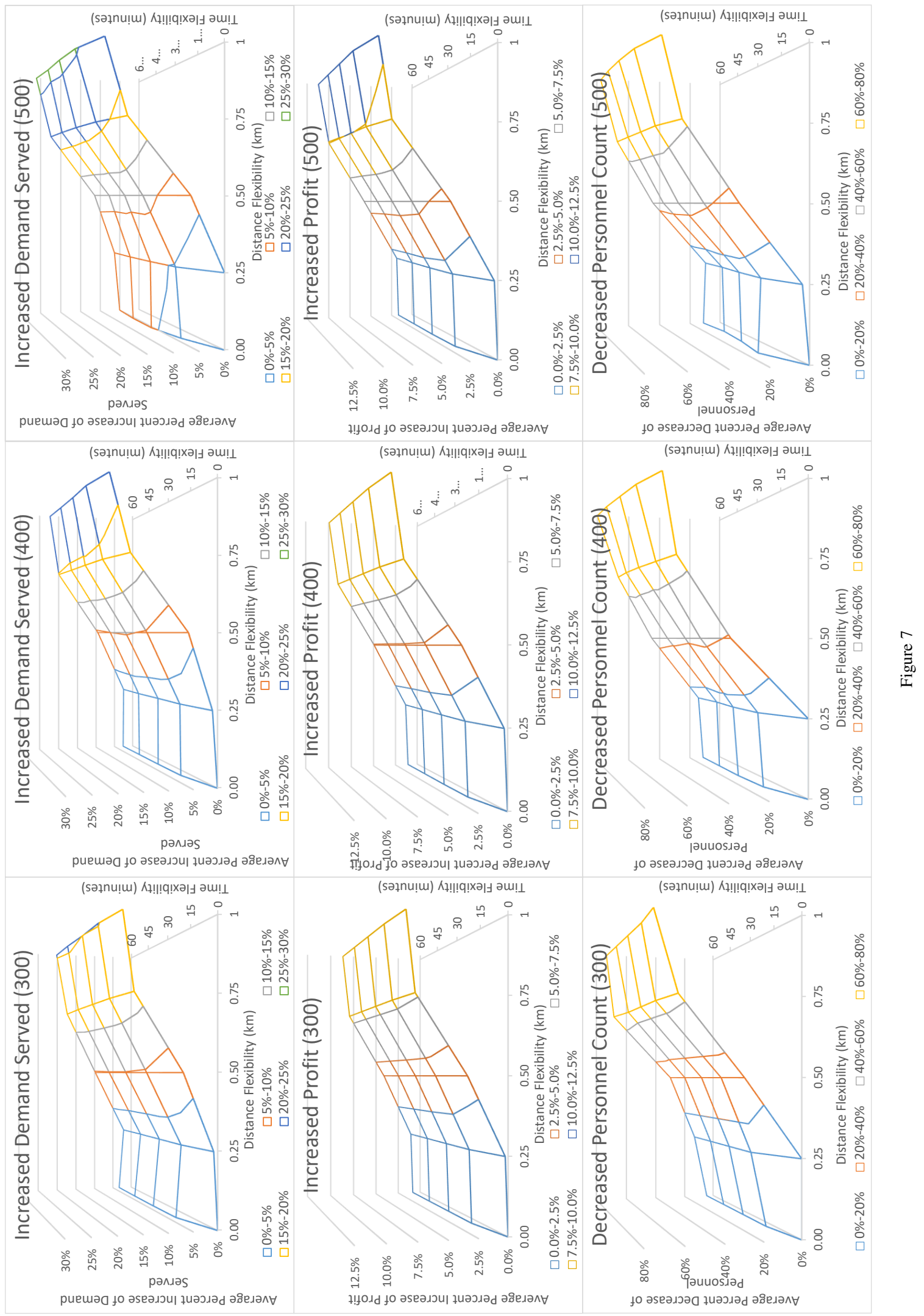




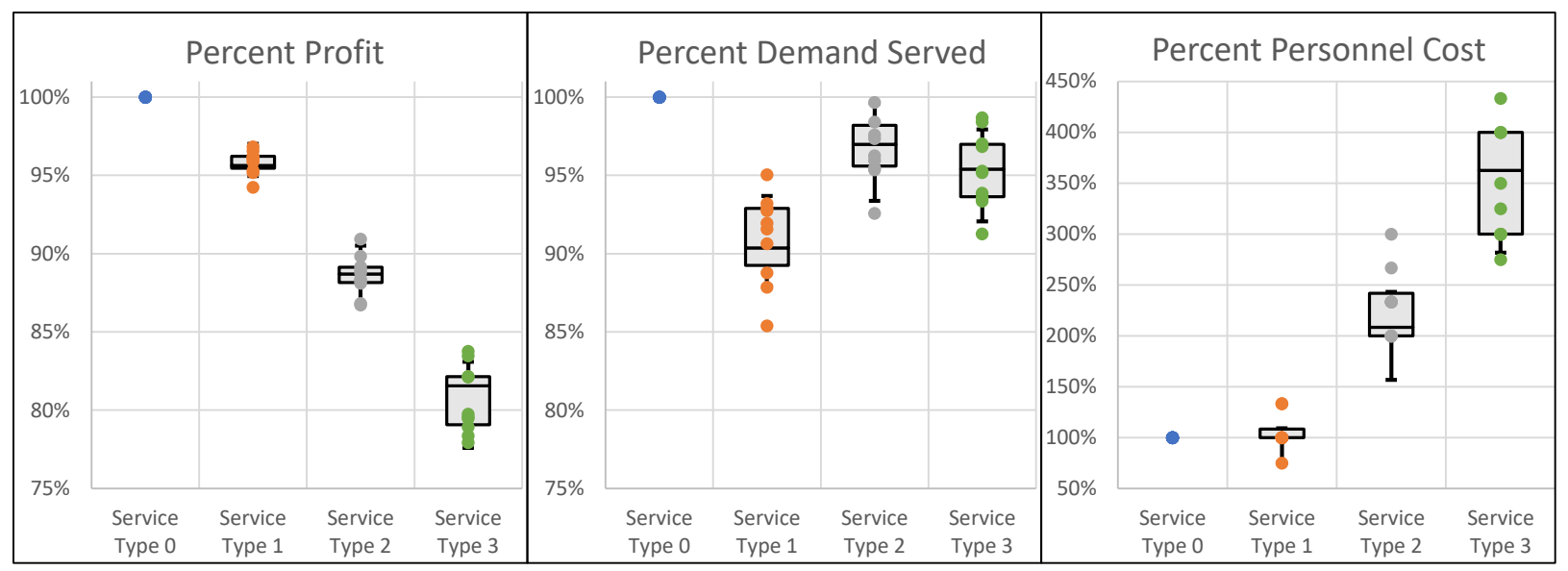

Figure 8

decided accordingly. In other words, the system works with perfect information. In Service Type 2 and 3, the system and eventually the solution framework processes the demand in batches. At every iteration 5\% of the demand requests are known to the system. The solution framework has to inform the users if it will be able to serve the requested demand or not. In Service Type 3, we assume that the system informs the users by providing the users the exact pick-up time, and origin and destination stations. On the other hand, in Service Type 2, the users are only informed if they are going to be served or not. They do not learn the pick-up time, and origin and destination stations until the entire set of demand requests are processed. If there are no temporal or spatial flexibility introduced to the system, Service Type 2 and 3 return the same results since there will be only one option to serve a demand. However, when there is flexibility introduced, systems providing Service Type 2 may use the opportunity of delaying decisions and may profit more or serve more requests. With this analyses, we want to demonstrate if there is any benefit in delaying these decisions. Fig. 8 depicts the effect of different service types to 10 different instances with 400 demand, 500 meters of spatial flexibility and 30 minutes of temporal flexibility. We compare all the runs with the instances run under the setting of the Service Type 0. Service Type 0 assumes vehicles do not need any charging and removes the safety gap between consecutive rentals.

The graph in the left of Fig. 8 compares the percent profits of each service type compared to the Type 0 . This figure shows that introducing the safety gap decreases the profit by around $4 \%$ on average. In addition, when the system is utilizing a configuration as Service Type 3, it loses around $15 \%$ of its profit. However, if the time and location information is delayed to the end of the decision process (see Service Type 2), $8 \%$ of this loss can be recovered.

The graph in the middle of Fig. 8 shows that, introducing safety gap to the model prevents us to serve around $10 \%$ more demand if the entire set of demand requests are known in advance. However, surprisingly, Type 2 and Type 3 Service configurations serve more demand. This is most probably because of the limited information about the incoming demand. The system operating with Service Type 1 accepts to serve fewer but more profitable demand requests whereas since the systems operating with Service Type 2 and 3 give their decisions about each demand request without knowing the incoming demand requests in the future, they choose more but less profitable demand requests.

The graph on the right shows how many personnel is used at each system configuration. As expected, the Service Type 0 uses the least personnel on average although there are instances of Service Type 1 using fewer personnel. We see the number of personnel more than doubles when the system is served by Service Type 2 . The number of personnel in Service Type 3 is the highest among the configurations. It needs on average 2.5 more personnel than the Type 0 case. The systems that are operated by Service Type 2 and 3 serving more demand but also using more personnel to maintain the service level of the system. 


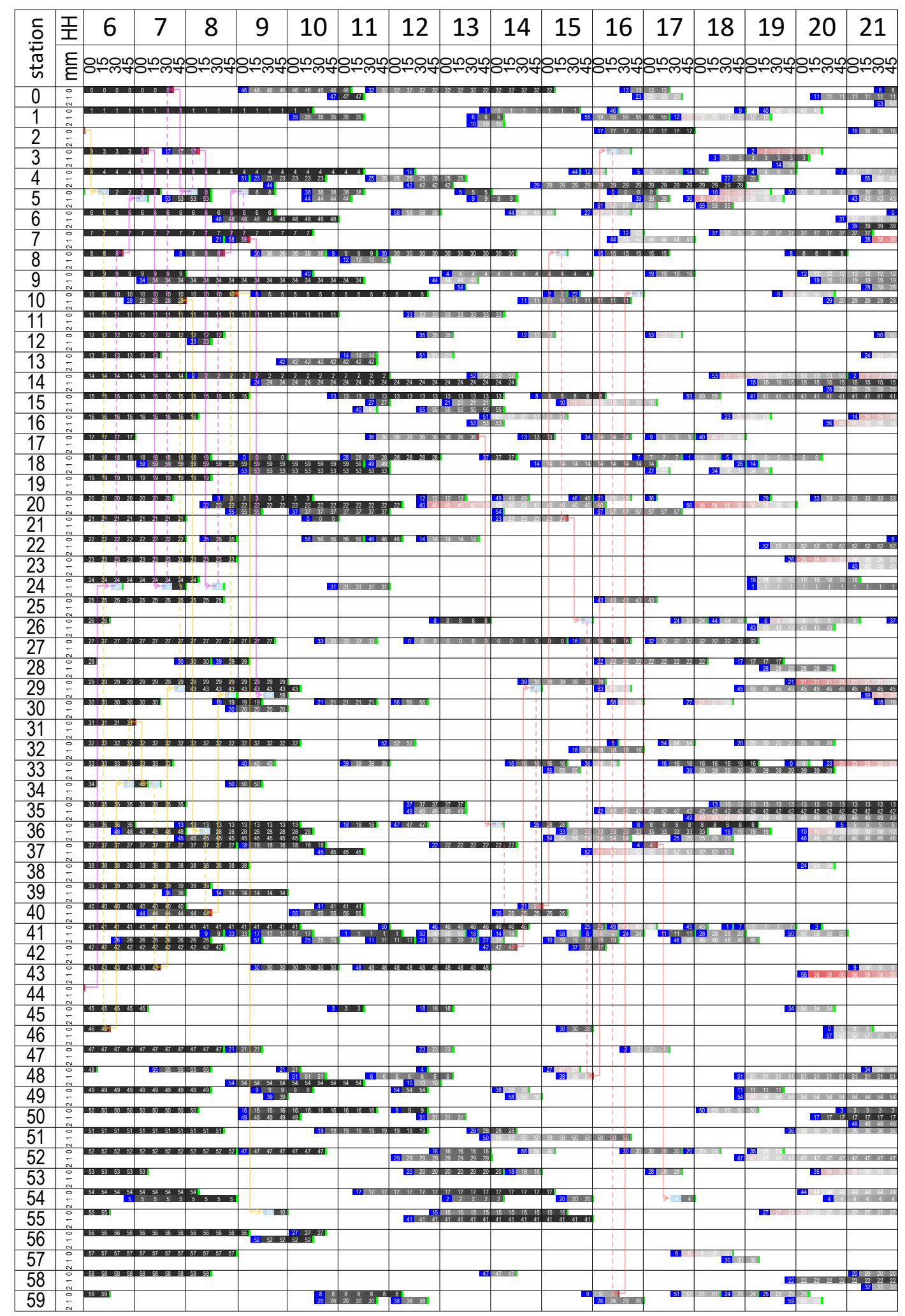

Figure 9: Schedule of vehicles, stations and relocation personnel for the selected instance with 500 demand (see Fig. 10 for the color coding). 


\begin{tabular}{|c|c|c|c|c|}
\hline & STATE & & STATE & 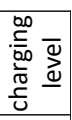 \\
\hline & empty spot & $\#$ & \multirow{4}{*}{$\frac{\frac{0}{0}}{\frac{\pi}{\pi}}$} & $100 \%$ \\
\hline$\#$ & arrival from trip & $\#$ & & $80 \%$ \\
\hline \# & arrival from relocation & \# & & $60 \%$ \\
\hline & departure for trip & \# & & $40 \%$ \\
\hline & departure for relocation & \# & \multirow{3}{*}{$\begin{array}{ll}\frac{1}{d} \\
\frac{1}{d} \\
\frac{0}{0} \\
\frac{0}{0} \\
\frac{0}{5}\end{array}$} & $30 \%$ \\
\hline$\longrightarrow$ & relocation with a vehicle & $\#$ & & $15 \%$ \\
\hline$--\rightarrow$ & relocation without a vehicle & \# & & 0 \\
\hline
\end{tabular}

Figure 10: Legend of Fig. 9

\subsection{Detailed decision and performance analyses of an instance}

The proposed framework also provides the operator, very specific information of which demand to accept/reject and offer as an alternative and how to schedule the relocation personnel. In this subsection we take one of the instances and analyze it with the tools provided by the solution framework. The aim of this analysis is to show the capabilities of the solution framework in making operational decisions.

In this section we analyze one of the instances with 500 demand requests with 1000 meters spatial and 60 minutes temporal flexibility. Among 500 demand requests, the operational framework decides to serve 352 of them with 3 personnel. We present the other outcomes of the system with a fig.s 9-12

Fig. 9 shows the state of each station throughout the day. It also presents the vehicle and personnel movements in details. The first and the second columns of the figure show the station and spot IDs for each station. The first and second row of the figure show the starting hour and minute of the time interval. Every station in the system has 3 parking spots. At the beginning of the day, there is one vehicle available at each station. In this specific instance, there were 3 personnel were assigned to the system. 2 of these personnel were assigned to the shift very early in the morning whereas the last personnel was working on the afternoon shift between 13:45-17:45. The movements of the personnel with (straight line) and without vehicle (dashed line) between stations are also shown in this figure. If the spot is occupied by a vehicle, its ID can be seen inside the colour coded boxes. The state of the vehicles are shown with different colours. If a vehicle is staying at a station, it is represented with either gray-black or gray-red colours. The former shows the vehicle has at least $40 \%$ charging level and available for a client to pick-up. As the vehicle charging level gets higher, the colour gets darker. The latter shows that the vehicle is currently undercharged and cannot be picked-up by a user. As the vehicle charging level gets lower, the colour gets redder. A vehicle can be picked-up from or dropped-off to a spot either by a personnel for a relocation operation (red or light blue) or a user for a trip (green or blue). The complete colour coding for the figure is also given in Fig. 10.

It can be seen from Fig. 9 that most of the vehicles have a charging level enough for a trip specifically at the beginning of the day. Towards the end of the day, we start to see more vehicles starting to get undercharged. Furthermore, we see that, there are a few stations with high vehicle occupations, e.g. stations 5, 9, 20 and 36, whereas most of the stations have never used more than one spot, e.g. stations 2, 11, 17, 19, 21, 45. Increasing the capacities of the former set of stations could help the system to serve more demand whereas if there is a need to remove a few spots, decreasing the sizes of the stations from the latter set could not effect the performance of the system in general.

Fig. 11 shows how the solution framework uses the spatial and temporal flexibility. The $\mathrm{x}$ and $\mathrm{y}$ axes show the distance between the requested and proposed origin and destination stations respectively. The different colours of the bubbles show if the demand is served on time (blue), 15 minutes earlier (gray) or 15 minutes later (orange) than the requested time. The figure shows among 352 served demand, 341 of them are served on time, 4 of them are served 15 minutes earlier and 7 of them are served 15 minutes later than requested. 230 demand served at their original pick-up time, from the requested origin and destination stations. All 4 demand served 15 minutes earlier served from the exact origin and destination stations. Among 7 demand served later, 5 of them are served from the exact origin and destination stations. The other two demand are dropped-off to stations different than the requested destination station. 111 demand requests are served on time from different origin and/or destination stations. Among these demand requests, only 11 of them are served from both different origin and different destination stations. Although this is the result of a single instance, it shows that although it is available, over $65 \%$ of the demand is served at the 


\section{Distribution of Demand Flexibility Offered}

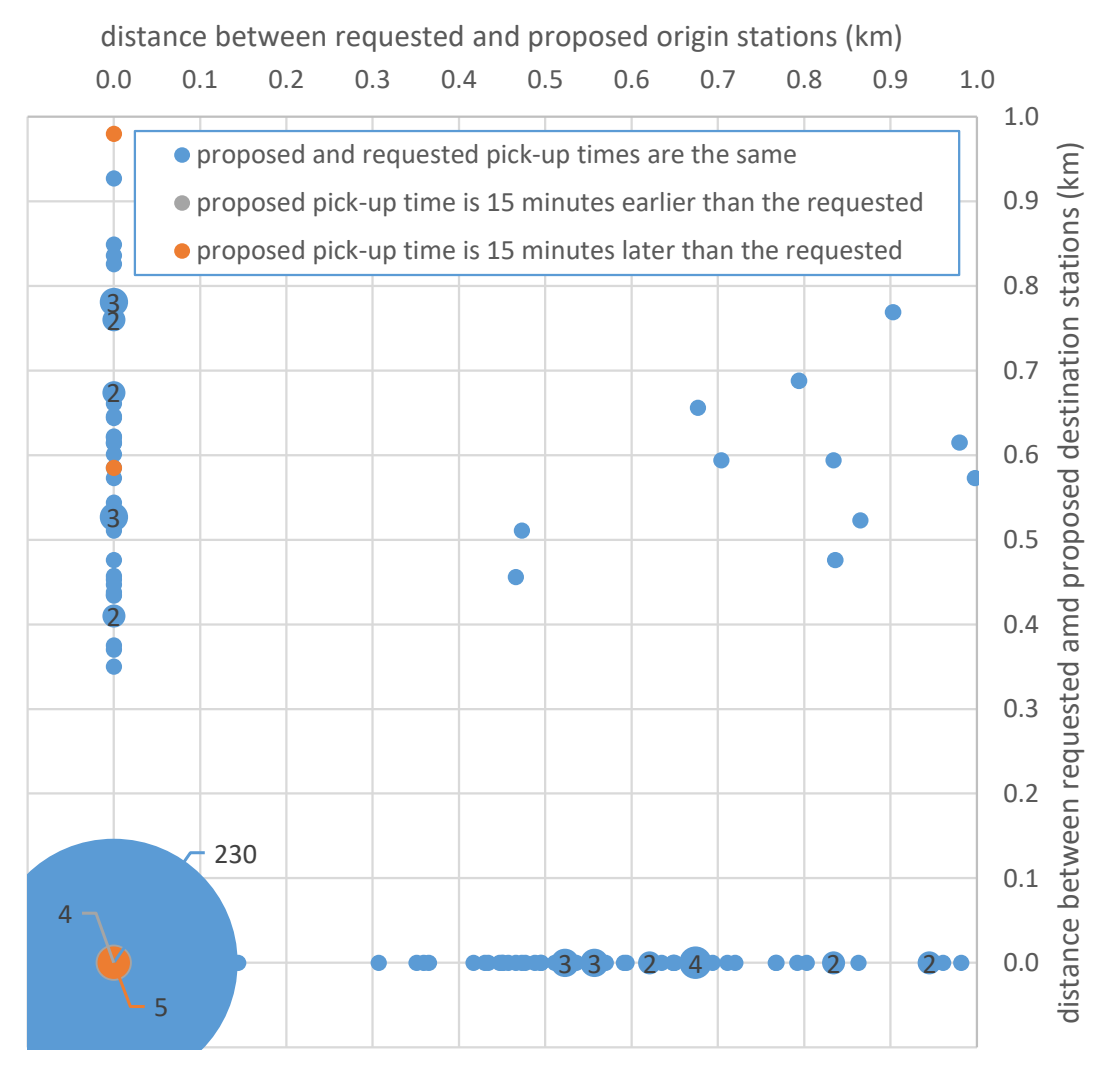

Figure 11: Bubble chart presenting the distance and time differences between the requested and served trip start times, and origin and destination stations.

requested time and stations. The solution framework does not prefer to use the flexibility option in most of the cases since it costs to the system.

Fig. 12 shows how each vehicle was utilized during the day. We see the vehicle IDs on x-axis. The vehicles are ordered according to their total utilization time. The number of trips served by each vehicle is also added to the figure, on top of the columns showing the total utilization of the vehicles. Average utilization of the vehicles were $45 \%$ and most of the vehicles utilized around this value. There are a few vehicles which are over- or under-utilized a lot. Vehicle number 31 is the most utilized vehicle with utilization level over $75 \%$. However it is also interesting that it serves only 3 trips. Further looking into data show that one of the trips (from station 50 to station 6 starting at 13:30 and ending at 21:00 it has served take 7.5 hours which makes almost $47 \%$ of vehicles utilization. Vehicle 9 serves maximum number of the trips. It has served 10 trips and relocated once.

\section{Concluding Remarks and Future Work}

In this paper we have introduced an integrated modeling and computational framework for analyzing the effect of spatial and/or temporal flexibility and reservation processing type on the performance of one way electric carsharing systems. The proposed framework consists of the following modules: preprocessing, optimization and simulation modules which model the one-way electric carsharing operations realistically in terms of the vehicle and personnel relocation, and battery charging requirements. The proposed framework was applied using the operational characteristics of a real system in Nice France, and by parametrically changing the spatial and temporal flexibility attributes of 


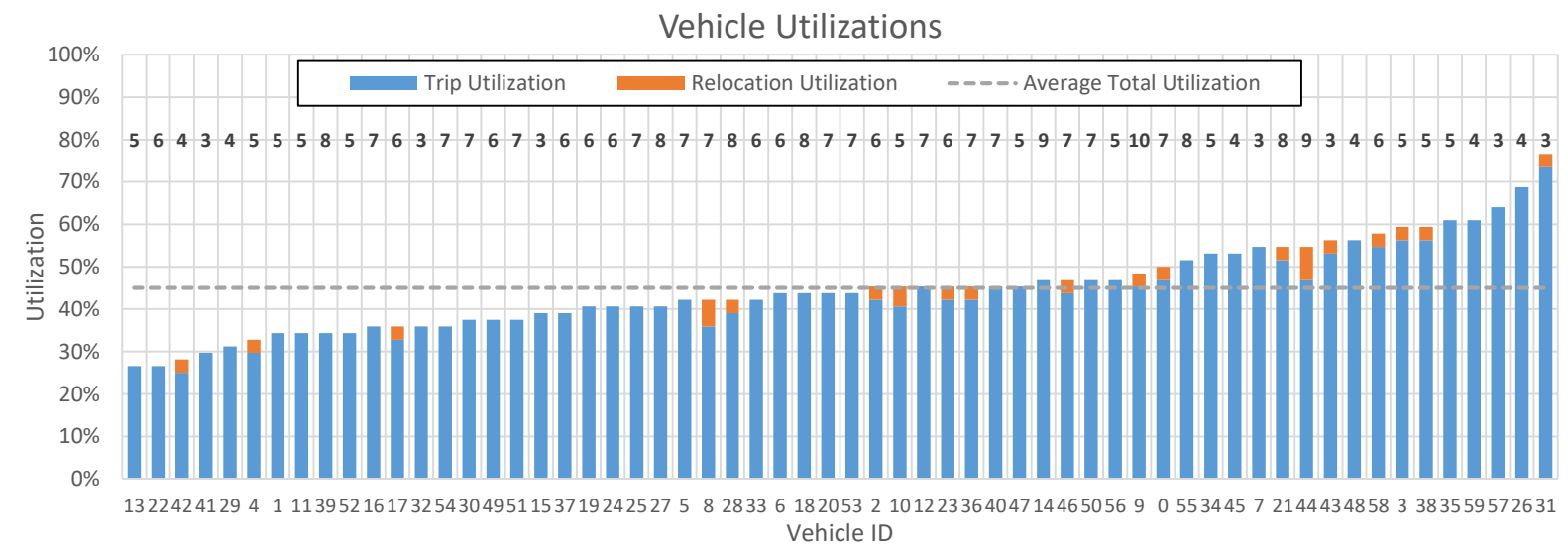

Figure 12: Utilization of vehicles. Numbers on top of the columns show the number of trips served by each vehicle.

the requested trips.

The proposed system provides the capability to analyze (1) the effect of spatial and temporal flexibility and (2) the effect of different service type on the system performance. In addition, the proposed framework is (3) capable to provide the best operational decisions that maximizes the total profit gained from the system for an instance of a one-way (electric) carsharing system.

For the specific case study analyzed we observe that the spatial flexibility has more significant effect than the temporal flexibility. Furthermore, for the system that we analyze, we observe that if the spatial flexibility is set to be 1000 meters, the system can increase its profit around $10 \%$ by serving over $25 \%$ more demand and hiring almost $80 \%$ fewer relocation personnel.

In our analyses, we set a fixed value and incentive for spatial and temporal flexibility provided by the system. We assume that every trip can be served from/to alternative stations at different pick-up times for the same incentives. However, in reality, the acceptable level and value of flexibility may differ according to user and trip types. Integrating behavioural models to the proposed framework could provide more accurate assumptions. Better assumptions could give more accurate solutions. To make these models more applicable to real life systems, integrating behavioural models to operational decisions should be a research priority. With better designed relocation operations and incentives given to the users for the temporal and flexibility they are willing to accept, one-way systems can be more profitable to the operators and accessible to users.

\section{References}

Auto Bleue, 2018. Accessed on 15/12/2018. https: //www . auto-bleue.org/en

Barth, M., Todd, M., Xue, L., 2004. User-based vehicle relocation techniques for multiple-station shared-use vehicle systems. Transportation Research Record: Journal of the Transportation Research Board 1887, 137-144.

Boyac1, B., Zografos, K. G., Geroliminis, N., 2015. An optimization framework for the development of efficient one-way car-sharing systems. European Journal of Operational Research 240 (3), 718-733. http://dx.doi.org/10.1016/j.ejor.2014.07.020.

Boyac1, B., Zografos, K. G., Geroliminis, N., 2017. An integrated optimization-simulation framework for vehicle and personnel relocations of electric carsharing systems with reservations. Transportation Research Part B 95, 214-237. http://dx.doi.org/10.1016/j.trb.2016.10.007.

Brandstätter, G., Gambella, C., Leitner, M., Malaguti, E., Masini, F., Puchinger, J., Ruthmair, M., Vigo, D., 2016. Overview of optimization problems in electric car-sharing system design and management. In: Dynamic perspectives on managerial decision making. Springer, pp. 441471. https://link.springer.com/chapter/10.1007/978-3-319-39120-5_24

Bruglieri, M., Colorni, A., Luè, A., 2014. The vehicle relocation problem for the one-way electric vehicle sharing: An application to the Milan case. Procedia - Social and Behavioral Sciences 111, 18-27, Transportation: Can we do more with less resources? - 16th Meeting of the Euro Working Group on Transportation - Porto 2013. http://dx.doi.org/10.1016/j.sbspro.2014.01.034.

Correia, G. H. D. A., Jorge, D. R., Antunes, D. M., 2014. The added value of accounting for users' flexibility and information on the potential of a station-based one-way car-sharing system: an application in Lisbon, Portugal. Journal of Intelligent Transportation Systems: Technology, Planning, and Operations 18 (3), 299-308. http://dx.doi.org/10.1080/15472450.2013.836928.

Crane, K., Ecola, L., Hassell, S., Natarah, S., 2012. An alternative approach for identifying opportunities to reduce emissions of greenhouse gases. Tech. rep., RAND Corporation. http://www.rand.org/content/dam/rand/pubs/technical_reports/2012/RAND_TR1170.pdf 
Ferrero, F., Perboli, G., Rosano, M., Vesco, A., 2018. Car-sharing services: An annotated review. Sustainable Cities and Society 37,501 - 518. http://dx.doi.org/10.1016/j.scs.2017.09.020.

Gambella, C., Malaguti, E., Masini, F., Vigo, D., 2018. Optimizing relocation operations in electric car-sharing. Omega 81 , 234 - 245. http://dx.doi.org/10.1016/j.omega.2017.11.007.

Google, 2018. Google Maps API. Accessed on 15/12/2018. https://developers.google.com/maps/

Jorge, D., Correia, G. H., 2013. Carsharing systems demand estimation and defined operations: a literature review. European Journal of Transport and Infrastructure Research 13 (3), 201-220, http://www.ejtir.tudelft.nl/issues/2013_03/pdf/2013_03_02.pdf.

Laporte, G., Meunier, F., Calvo, R. W., 2018. Shared mobility systems: an updated survey. Annals of Operations Research 271 (1), $105-126$. http://dx.doi.org/10.1007/s10479-018-3076-8.

Nair, R., Miller-Hooks, E., 2011. Fleet management for vehicle sharing operations. Transportation Science 45 (4), 524-540. http://dx.doi.org/10.1287/trsc.1100.0347.

Nourinejad, M., Zhu, S., Bahrami, S., Roorda, M. J., 2015. Vehicle relocation and staff rebalancing in one-way carsharing systems. Transportation Research Part E: Logistics and Transportation Review 81, 98-113. http://dx.doi.org/10.1016/j.tre.2015.06.012.

Repoux, M., Boyac1, B., Geroliminis, N., 2015. Simulation and optimization of one-way car-sharing systems with variant relocation policies. In: 94th Annual Meeting of the Transportation Research Board. No. 15-1907. Washington D.C., p. 18. http://trid.trb.org/view.aspx?id= 1337340

Shaheen, S. A., Chan, N. D., Micheaux, H., 2015. One-way carsharing's evolution and operator perspectives from the Americas. Transportation 42 (3), 519-536. http://dx.doi.org/10.1007/s11116-015-9607-0.

UN, 2014. World urbanization prospects: The 2014 revision-highlights. Accessed on 15/12/2018. https://esa.un.org/unpd/wup/ publications/files/wup2014-highlights.pdf

$\mathrm{Xu}$, M., Meng, Q., Liu, Z., 2018. Electric vehicle fleet size and trip pricing for one-way carsharing services considering vehicle relocation and personnel assignment. Transportation Research Part B: Methodological 111, 60 - 82. http://dx.doi.org/https://doi.org/10.1016/j.trb.2018.03.001.

Zhao, M., Li, X., Yin, J., Cui, J., Yang, L., An, S., 2018. An integrated framework for electric vehicle rebalancing and staff relocation in one-way carsharing systems: Model formulation and lagrangian relaxation-based solution approach. Transportation Research Part B: Methodological 117, 542-572. http://dx.doi.org/10.1016/j.trb.2018.09.014.

\section{Appendix A. Vehicle Assignment Model}

Although the VAS returns charging level feasible vehicle assignments in most of the instances, it is not exploring the entire solution space and may not be able to find an existing feasible solution. In those instances, we can use the JFM to find a feasible solution. However, an intermediary model which is faster than JFM but more accurate than VAS could be useful. For this reason, we developed another model that uses the relocations created by the PFM instead of deciding everything in one model as JFM does.

The VAM assigns vehicles to already settled flows. It takes all the rental flows from the FOOM and all driving flows from the PFM, and finds flows for each vehicle. When the flow assignment is being done, the VAM keeps track of the charging levels of each vehicle. The objective function aims to minimize the number of infeasible flows existing in the model. These flows that are identified by the VAM are added to set $C$. The FOOM is solved again with the updates in $C$ to find a charging level feasible solution.

$\min \sum_{k, f} \tilde{c}_{k f}$

subject to

$\sum_{k} \tilde{y}_{k f}=1$

$\forall f \in F(\mathrm{~A} .2)$

(23), (25), (31) - (35), (37)

The VAM aims to serve the trip and relocation flows identified by the FOOM and PFM respectively while minimizing the number of infeasible flows (Objective A.1). Different than the flows in the PFM or JFM, all the flows including the relocation flows in this model are all originated from and destined to stations. They are not from and/or to cluster nodes. Strictly speaking, the flow the VAM deals with are real complete flows between two stations. That is the reason, we introduce constraints A.2 to the model instead of using Constraints 29 and 30 . Note that, the set $F$ in this new model is composed of complete vehicle flows only. That is the reason, constraints A.2 should hold for all flows.

Unfortunately, our experiments have shown that the VAM is solved on average less than one minute faster than the JFM for the same instances. Although its accuracy is slightly lower than JFM, we decided it is better to remove the VAM from the solution process and use the JFM if the VAS cannot produce a feasible solution. 